\title{
COMPARISON BETWEEN PATH LENGTHS TRAVELED BY SOLAR ELECTRONS AND IONS IN GROUND-LEVEL ENHANCEMENT EVENTS
}

\author{
Lun C. Tan ${ }^{1,2}$, Olga E. Malandraki ${ }^{2}$, Donald V. Reames ${ }^{3}$, Chee K. $\mathrm{NG}^{4}$, Linghua Wang ${ }^{5,6}$, \\ IoAnna Patsou ${ }^{2}$, And Athanasios Papaioannou ${ }^{2}$ \\ ${ }^{1}$ Department of Astronomy, University of Maryland, College Park, MD 20742, USA; 1tan@ umd.edu \\ ${ }^{2}$ Institute of Astronomy and Astrophysics, Space Applications and Remote Sensing, National Observatory of Athens, Athens, Greece \\ ${ }^{3}$ Institute for Physical Science and Technology, University of Maryland, College Park, MD 20742, USA \\ ${ }^{4}$ College of Science, George Mason University, Fairfax, VA 22030, USA \\ ${ }^{5}$ Department of Geophysics, Peking University, Beijing 100871, China \\ ${ }^{6}$ Space Science Laboratory, University of California, Berkeley, CA 94720, USA \\ Received 2012 September 4; accepted 2013 March 9; published 2013 April 16
}

\begin{abstract}
We have examined the Wind/3DP/SST electron and Wind/EPACT/LEMT ion data to investigate the path length difference between solar electrons and ions in the ground-level enhancement (GLE) events in solar cycle 23. Assuming that the onset time of metric type II or decameter-hectometric (DH) type III radio bursts is the solar release time of non-relativistic electrons, we have found that within an error range of $\pm 10 \%$ the deduced path length of low-energy $(\sim 27 \mathrm{keV})$ electrons from their release site near the Sun to the $1 \mathrm{AU}$ observer is consistent with the ion path length deduced by Reames from the onset time analysis. In addition, the solar longitude distribution and IMF topology of the GLE events examined are in favor of the coronal mass ejection-driven shock acceleration origin of observed non-relativistic electrons. We have also found an increase of electron path lengths with increasing electron energies. The increasing rate of path lengths is correlated with the pitch angle distribution (PAD) of peak electron intensities locally measured, with a higher rate corresponding to a broader PAD. The correlation indicates that the path length enhancement is due to the interplanetary scattering experienced by first arriving electrons. The observed path length consistency implies that the maximum stable time of magnetic flux tubes, along which particles transport, could reach $4.8 \mathrm{hr}$.
\end{abstract}

Key words: acceleration of particles - Sun: coronal mass ejections (CMEs) - Sun: flares Sun: particle emission - Sun: radio radiation

Online-only material: color figures

\section{INTRODUCTION}

\subsection{Motivation of the Investigation}

One of the fundamental questions in solar energetic particle (SEP) investigation is the acceleration mechanism of the highest energy (GeV and above) particles (Cliver 2008). Ions at these energies may interact in Earth's atmosphere to produce secondary particles of sufficient intensity that can be detected by neutron monitors at ground level, causing the ground level enhancement (GLE) event. Since high-energy ions represent "hard" radiation that can be a significant hazard to astronauts and equipment in space, while secondary neutrons threaten passengers and crew of aircraft on polar routes, understanding where, when, and how particle acceleration takes place in the GLE event is also an important issue in space weather forecasting (Reames 2009a, 2009b). Taking into account typical ion energy spectra and the thickness of available shielding, the most important energy range of protons in space radiation damage is from 30 to 100-200 MeV (Turner 2006).

It is generally accepted that solar ions in GLE events are accelerated by the coronal mass ejection (CME)-driven shock waves, while the remnant suprathermal ions from previous SEP events may contribute to the seed population of shockaccelerated particles (Tylka \& Lee 2006). The onset time analysis, which is based on the scatter-free transport assumption of first arriving particles, is often used to calculate the solar release time (SRT) and path length $\left(L_{0}\right)$ that particles traveled from their release site near the Sun to the observer (e.g.,
Reames 2009a, 2009b). However, there has been a long-lasting divergence between $L_{0}$ values deduced from solar electron and ion data (e.g., Tylka et al. 2003; Mewaldt et al. 2003). The divergence might imply that the solar release site of electrons is different from that of ions. However, the divergence could be also due to particle transport effects; in particular, the transport of the first arriving electrons may not be scatter-free (see later Section 1.4.2). Recently, theoretical studies (Saiz et al. 2005; He et al. 2011; Rouillard et al. 2012) have shown the complexity of onset time analysis. Obviously, care should be taken to resolve the $L_{0}$ divergence between solar electrons and ions.

Without depending on the onset time analysis, Larson et al. (1997) and Kahler et al. (2011a, 2011b) calculated the $L_{0}$ value of electrons in the impulsive electron event occurring in a magnetic cloud (MC) by assuming that (1) these electrons are released at the onset time of type III radio bursts (RBs), (2) the interplanetary (IP) transport of first arriving electrons is scatter-free. Their approach provides a new way to decouple SRT deduction from the $L_{0}$ calculation, motivating us to adopt a similar way to estimate the $L_{0}$ value of solar electrons in the GLE event.

\section{2. "Impulsive" Solar Particle Events}

Small non-relativistic solar electron events appear to be the most common type of impulsive particle emission from the Sun (see the review of Lin 1985). Impulsive electron events were first observed at the electron energy $E_{e}>40 \mathrm{keV}$ with a fast rise-slow decay time profile indicating the presence of significant particle 
scattering in the interplanetary medium (IPM). Later, the events were detected down to $E_{e} \sim 1 \mathrm{keV}$ (and even $E_{e} \sim 0.1 \mathrm{keV}$; see Gosling et al. 2003) with a fast rise-fast decay time profile indicating an essentially scatter-free propagation in the IPM (Lin 1974). In a few cases impulsive electron events are accompanied with impulsive ion events that exhibit higher ${ }^{3} \mathrm{He} /{ }^{4} \mathrm{He}$ and $\mathrm{Fe} / \mathrm{O}$ ratios (so-called ${ }^{3} \mathrm{He}$-rich event) and higher ionization status of Fe ions (Reames 1999).

Being different from the large "gradual" solar particle event that is attributed to the particle acceleration by CMEdriven shocks, the impulsive particle event is linked to the reconnection-driven acceleration in solar flares. In particular, impulsive events are associated with intense fast-driving decameter-hectometric (DH) type III RBs, while gradual events are accompanied with slow-drifting metric type II RBs (see the review of Cliver 2008).

As an example of impulsive solar electron events, Wang et al. (2006) examined the 1999 August 7 event by using the Wind $/ 3 \mathrm{DP}$ electron data of $E_{e}=0.4-310 \mathrm{keV}$. They noted that the observed time profiles of electron intensities at all energies fit well to triangular injections at the Sun with equal rise and fall times. At $E_{e}>25 \mathrm{keV}$ the full width of half-maximum (FWHM) of injection electrons is $<6$ minutes. In addition, they found that at $E_{e}>13 \mathrm{keV}$ the solar release time of electrons $\left(\mathrm{SRT}_{e}\right)$ is $7.6 \pm 1.3$ minutes later than the onset time $\left(t_{\mathrm{III}}\right)$ of type III RBs, whereas Krucker et al. (2007) observed a group of promptly arrived events with zero delay. Furthermore, by using the highresolution interplanetary magnetic field (IMF) data from the Wind and Advanced Composition Explorer (ACE) spacecraft, Tan et al. (2011) examined the power spectral density (PSD) of the IMF in the 1999 August 7 event. Near and above the proton gyrofrequency they observed a steepening of PSD spectra due to the damping of electromagnetic ion cyclotron (EMIC) waves by solar-wind thermal ions. The steepening significantly reduces the PSD level above the proton gyrofrequency, preferring the occurrence of the scatter-free transport of low-energy electrons during a time period longer than $5 \mathrm{hr}$. Consequently, in the $E_{e}$ range of 65-100 keV there appears to be an electron energy window, across which the scatter-free transport of lower energy electrons would change to the diffusive transport of higher energy electrons.

It is evident that through the open magnetic field lines involved in the reconnection process, solar particles accelerated in impulsive events may escape into the IPM and be detected by the $1 \mathrm{AU}$ observer. Because of the small spatial extent of the reconnection region the involved field lines should be confined into a narrow $\left( \pm 20^{\circ}\right)$ solar longitude cone centered at the well-connected longitude $\left(\sim \mathrm{W} 55^{\circ}-60^{\circ}\right.$; see Reames 2002). Recently, observations from the STEREO and ACE spacecraft (Wiedenbeck et al. 2011) showed that an impulsive particle event can be seen over a large longitudinal angle of $\sim 136^{\circ}$. However, since the $2-4 \mathrm{MeV}$ nucleon ${ }^{-1}{ }^{3} \mathrm{He}$ ions and $70-100 \mathrm{keV}$ electrons observed at the poorly connected angle begin to arrive some $\sim 16$ and $\sim 4 \mathrm{hr}$ after the well connected onset, respectively, these particles should not be on the field lines directly connected to the flare. Instead, the large longitude spread of these particles could be due to slow processes, which need to be further studied. As pointed out by Wiedenbeck et al. (2011), "Observations of energetic particle flux "dropouts" in rich SEP events (Mazur et al. 2000) have demonstrated that diffusive transport of energetic particles transverse to the heliospheric magnetic field is inefficient at distributing the particles in heliographic longitude. Thus, we discount this as a possible mechanism for producing the large longitudinal spread that we observe." In fact, the "drop-outs" of energetic particle intensities as observed by Mazur et al. (2000) and Chollet \& Giacalone (2011) in the impulsive particle event have shown that the gap boundaries of arriving particles to be extremely sharp, indicating that these particles do not experience any significant cross-field transport. Reames (2013) hence suggested that the longitude spread of solar particles is due to a perpendicular transport process by which energetic particles must travel a long distance before finding a crossover point produced by the field-line random walk of Jokipii \& Parker (1969). Note that the process is perpendicular transport, not perpendicular diffusion, because particles do not spread laterally at each radius. They only fill each flux tube, finding isolated crossover points at different radii to fill the next flux tube and eventually explore every nook and cranny of the field network. As a result, the particles from the impulsive particle events would be substantially delayed and attenuated when they spread to distant longitudes as observed by Wiedenbeck et al. (2011).

\subsection{Current Status of Path Length Measurements of Solar Particles}

Here we first mention the apparent inconsistency of $L_{0}$ measurements between Tylka et al. (2003) and Mewaldt et al. (2003). Tylka et al. (2003) carried out the onset time analysis of two large impulsive and three western GLE events by using the data of 100-300 keV electrons and $\sim 2 \mathrm{MeV}$ nucleon $^{-1}$ to $\sim 2 \mathrm{GeV}_{\text {nucleon }}{ }^{-1}$ ions from Wind, $A C E$, and IMP-8, as well as available neutron monitors. They found that the onset time analysis of electrons matches that of ions, implying that $\mathrm{SRT}_{e} \sim$ $\mathrm{SRT}_{i}$ and $L_{0 e} \sim L_{0 i}$. Hereafter, the subscripts $e$ and $i$ are used to denote electron and ion, respectively. In contrast, Mewaldt et al. (2003) examined 11 SEP events with enough intensity of the charge number $Z \geqslant 6$ ions by using $38-315 \mathrm{keV}$ electron and 6-88 MeV nucleon ${ }^{-1}$ ion data from the SIS and EPAM sensors on $A C E$, respectively. They found that only in four events, in which the $5-13 \mathrm{MeV}$ nucleon ${ }^{-1}{ }^{3} \mathrm{He} /{ }^{4} \mathrm{He}$ ratio is $>0.02$, do electrons and ions have similar SRT and $L_{0}$ values.

Compared with the onset time analysis of solar ions, the analysis of solar electrons displays more uncertainties. In the earlier works, $\mathrm{SRT}_{e}$ is deduced by assuming $L_{0 e} \sim 1.2 \mathrm{AU}$ (Haggerty \& Roelof 2002; Haggerty et al. 2003) or the length of the Parker spiral line under the observed solar wind speed (Krucker et al. 1999), which may result in errors. We hence mainly refer to Kahler \& Ragot (2006), who carried out the onset time analysis of 80 near-relativistic solar electron events observed by Wind/3DP/SST. The $L_{0 e}$ values deduced from the electron velocity dispersion relation are broadly distributed between 0.15 and $2.7 \mathrm{AU}$. In most cases $L_{0 e}<1 \mathrm{AU}$ with a median value of $0.88 \pm 0.09 \mathrm{AU}$, which is an unphysical result for the $1 \mathrm{AU}$ observer. Also, they examined 23 separate Wind/3DP/EESA electron events at lower energies (down to $\left.E_{e} \sim 1 \mathrm{keV}\right)$ and 24 combined SST+EESA events. For the separate EESA and combined SST+EESA events the median values of $L_{0 e}$ are $0.95 \pm 0.07 \mathrm{AU}$ and $0.99 \pm 0.06 \mathrm{AU}$, respectively, which are higher than the $L_{0 e}$ value of SST events at higher energies.

In addition, during an MC event on 1995 October 18-20, Larson et al. (1997) observed five impulsive electron events. Assuming that solar electrons are released at the onset time of type III RBs (i.e., $\mathrm{SRT}_{e}=t_{\mathrm{RB}}$, where $\mathrm{RB}=\mathrm{III}$ ) they found that the $L_{0 e \text { RB }}$ (i.e., $L_{0 e I I I}$ ) value varies from $\sim 3.2 \mathrm{AU}$ near the 
MC exterior to $\sim 1.2$ AU near the MC center. Nevertheless, no more impulsive electron events with $L_{0 e I I I} \geqslant 3.2 \mathrm{AU}$ have been observed since Larson et al. (1997), even though an extensive search for such event in MCs (Kahler et al. 2011b) and interplanetary coronal mass ejections (Kahler et al. 2011a) has been completed.

\subsection{Possible Errors Involved in Electron Path Length Measurements}

\subsubsection{Lack of Deposition Energy Loss Correction of Wind/3DP/SST Electron Data}

Since in the Wind/3DP/EESA-H data the instrumental noise mainly appears in the electron energy $E_{e}=4-25 \mathrm{keV}$ range (Wang et al. 2006), we restrict ourselves to analyzing the Wind $/ 3 \mathrm{DP} / \mathrm{SST}$ electron data in the $E_{e}=27-510 \mathrm{keV}$ range. Note that in the SST data $\sim 15 \%$ of incident electrons scatter out of the silicon detector and leave only a fraction of $E_{e}$ in it, producing an approximately even spectrum below $E_{e}$ (Berger et al. 1969; Haggerty \& Roelof 2002; Wang et al. 2011), which could imitate an earlier arrival of lower-energy electrons and produces a smaller $L_{0 e}$ value from the onset time analysis. Since the SST data downloaded from the 3DP Web site (http://sprg.ssl.berkeley.edu/wind3dp/) have not included the deposition energy loss correction, care should be taken when these data are used in the onset time analysis. As demonstrated below, the use of uncorrected SST data could result in an unphysical result of $L_{0 e}$ estimations. Therefore, in this work we have only used the SST data with deposition energy loss correction.

\subsubsection{Interplanetary Transport of First Arriving Electrons May Not be Scatter-free}

It is known (e.g., Reames 2009a, 2009b) that the onset time analysis of solar particles is based on the assumption that the particles that arrive first at the observer should experience scatter-free transport, with their pitch-angle $(\alpha)$ cosine value $(\mu=\cos (\alpha))$ of $\sim 1$. This assumption sounds reasonable in the analysis of solar ion data, because in large SEP events the pre-event background level of ion intensity is usually low. The magnetohydrodynamic (MHD) waves that scatter ions are mostly self-excited ( $\mathrm{Ng}$ et al. 2003). However, the situation may be different in the analysis of solar electrons. Because of the substantial presence of background electrons, usually both $R$-mode (whistler) and $L$-mode (EMIC) waves (Tan et al. 2011) that scatter non-relativistic electrons may already be amplified along the IP transport route of electrons. As a result, the IP transport of first arriving electrons may not be scatter-free.

In addition, in the SST measured $E_{e}$ range $(27-510 \mathrm{keV})$ Tan et al. (2011) observed the presence of an electron energy window, across which the electron scattering status would change from scatter-free at lower energies to diffusive at higher energies. While the location of the window is event-dependent, it is evident that in the $E_{e}$ range of SST that the IP scattering of electrons should be increased with increasing $E_{e}$, which is different from the ion transport in which the IP scattering of ions is decreased with increasing ion rigidity (energy; Jokipii 1971). As a result, it is necessary to check the real scattering status, in particular for electrons at higher energies $\left(E_{e}>65 \mathrm{keV}\right.$; see Tan et al. 2011, 2012).

Also, Tan et al. (2011) noted that the change of scattering status for higher energy electrons should occur in the local environment (the heliocentric distance $r>0.5 \mathrm{AU}$ ) because of the dominant focused transport effect of SEPs at smaller $r$ (see He \& Wan 2012 and references therein). The notion of Tan et al. (2011) is consistent with that of Wang et al. (2011), who observed that in their examined impulsive electron events the time profiles of electron intensities retain a rapid-rise, rapiddecay peak and the estimated path length is only $\sim 4 \%-18 \%$ longer than the length of the Parker spiral line, indicating that electron scattering has indeed occurred near 1 AU. Therefore, the analysis of locally measured PAD of electrons could be helpful to understand the average scattering behavior of solar electrons along their entire IP transport route.

\subsubsection{Temporal Variation of Magnetic Field Topologies}

Because of the sampling time difference between electrons and ions there could be a "real" inconsistency between $L_{0 e}$ and $L_{0 \mathrm{i}}$ if temporal variation of magnetic field topologies occurred during the examined event period. For example, Tan et al. (2012) observed that in the 2002 April 21 MC event the magnetic field topology seen by the Wind spacecraft during the first hour since the event onset is different from that during the later time, leading to $L_{0 e}=1.1 \pm 0.1 \mathrm{AU}$ being different from $L_{0 i}=$ $1.49 \pm 0.05 \mathrm{AU}$ based on the onset time analysis. Therefore, care should be taken to check the temporal variation of magnetic field topologies during the examined event period.

\subsection{Questions to be Addressed in This Work}

Using solar ion data from Wind, IMP-8, GOES, and neutron monitors, based on the onset time analysis Reames (2009a) deduced the $L_{0 i}$ value of the GLE events during solar cycle 23, which provides a fiducial mark of ion path lengths to be compared with electron observations. We hence wish to calculate the $L_{0 e}$ value for those GLE events in order to compare it with the $L_{0 i}$ value deduced by Reames (2009a). However, before doing such comparison we need to answer a few questions.

1. What is the effect of deposition energy loss correction on Wind/3DP/SST electron data?

Since the deduction of $L_{0 e}$ is heavily dependent upon the quality of SST electron data, we need to compare the $L_{0 e}$ values deduced from the SST data with and without the deposition energy loss correction, in order to check whether or not the deposition energy loss corrected $L_{0 e}$ value could be consistent with the $L_{0 i}$ value deduced by Reames (2009a).

2. What type of RBs should be associated with the nonrelativistic electrons observed in GLE events?

Since GLE events are characteristically linked with both big flares and fast CMEs, it is difficult to isolate the RB signatures associated with them (Cliver 2008). Therefore, in our further description we assume $\mathrm{RB}=\mathrm{II}$, although the replacement of $\mathrm{RB}=$ II by $\mathrm{RB}=\mathrm{III}$ does not alter our conclusion. Later in Section 3, however, we will explain why additional observational evidences prefer $\mathrm{RB}=\mathrm{II}$.

3. What is the electron energy at which a valid electron path length can be deduced?

Since in Larson et al. (1997) and Kahler et al. (2011a, 2011b) $L_{0 e}$ is calculated at individual $E_{e}$ channels of incident electrons, it is necessary to clarify at what $E_{e}$ value the deduced $L_{0 e}$ value is valid. As mentioned above, Tan et al. (2011) noted that in the $E_{e}$ range of SST the IP scattering of non-relativistic electrons would increase with 
increasing $E_{e}$. Consequently, the $L_{0 e \text { RB }}$ value estimated at the lowest $E_{e}$ value of SST should be close to the real value.

4. How can we accomplish the comparison between $L_{0 i}$ and $L_{0 e \mathrm{RB}}$ ?

In order to compare $L_{0 i}$ with $L_{0 e \mathrm{RB}}$, the metric and $\mathrm{D}-\mathrm{H}$ type II and III RB data as given in the Solar Geophysical Data (SGD) and by the Wind/WAVES instrument (Bougeret et al. 1995) are important. However, the RB data in SGD are often unreliable (Cane et al. 2010). Gopalswamy et al. (2012) wrote, "In some cases, discerning type II bursts from other emissions felt like an art rather than science." Thus, they also examined the actual dynamic spectra from individual observatory Web sites (Hiraiso, Culgoora, IZMIRAN, Nancay), and from the Radio Solar Telescope Network (RSTN). In all cases, they were able to check the dynamic spectra and hence verify the onset times of metric type II RBs within a few minutes. Therefore, in order to enhance the reliability, our analysis includes the use of three independent sources of observations: $L_{0 i}$ data from Reames (2009a), RB data from Gopalswamy et al. (2012), and $L_{0 e}$ data from this work. As a result, to a maximum extent our analysis could avoid the lack of experiences in any branch of involved analysis works.

The comparison of thus deduced $L_{0 e \mathrm{RB}}$ value with the $L_{0 i}$ value deduced by Reames (2009a) is presented in Section 2 and discussed in Section 3.

\section{OBSERVATIONS}

\subsection{Observed Data}

In this work, we use the Wind/3D Plasma and Energetic Particle Investigation (3DP; see Lin et al. 1995)/Semi-Conductor Detector Telescope (SST) electron data and the Wind/Energetic Particle Acceleration, Composition, and Transport Experiment (EPACT; see von Rosenvinge et al. 1995)/Low Energy Matrix Telescope (LEMT) heavy ion data. As explained above, the properties of RBs, flares, and CMEs for the GLE events during solar cycle 23 are taken from the compilation of Gopalswamy et al. (2012). Hereafter, the light-travel time of 8.3 minutes from the Sun to the Earth has been subtracted from the electromagnetic radiation observation time at $1 \mathrm{AU}$.

\subsection{Effect of Deposition Energy Loss Correction for Wind/3DP/SST Electron Data}

Based on the technique developed in Tan et al. (2009), we carry out the electron onset time analysis of the GLE events listed in Table 1 of Reames (2009a), using the uncorrected SST data that are directly downloaded from the 3DP Web site. In the onset time analysis, the observed time $t$ of first arriving particles is plotted as a function of the particle velocity $v$,

$$
t=t_{0}+L_{0} / v
$$

from which the solar release time $\left(\mathrm{SRT}=t_{0}\right)$ and the path length $\left(L_{0}\right)$ traveled by first arriving particles from their release site near the Sun to the observer can be calculated. Among the 13 GLE events listed in Table 1 of Reames (2009a) there is a lack of SST data in the 2006 December 13 event. Also, we are not able to complete the analysis of the 2003 October 29 event because of the high intensity of background electrons coming from an earlier event.

For the remaining 11 GLE events based on the onset time analysis from the uncorrected SST electron data the deduced

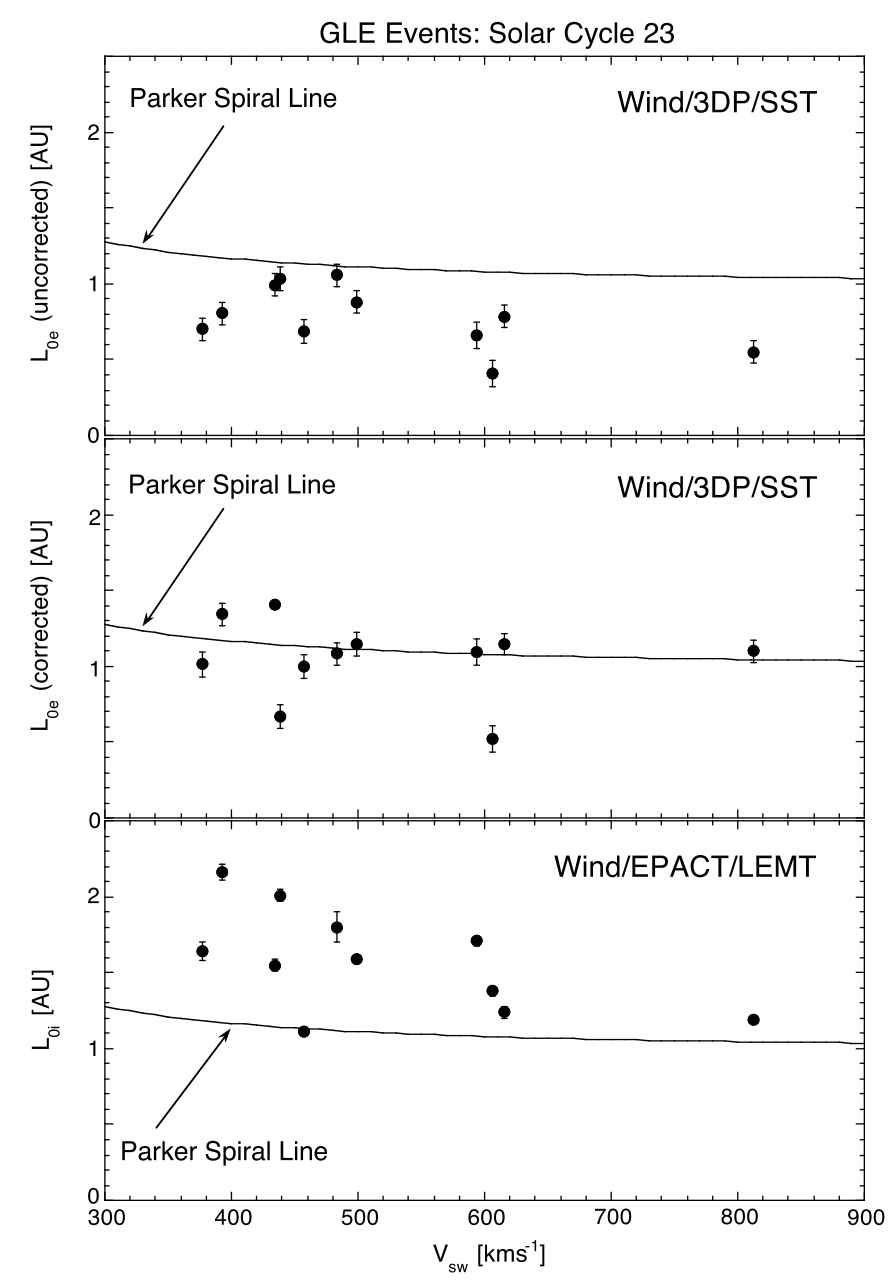

Figure 1. Particle path lengths deduced from the onset time analysis are shown as a function of the simultaneously measured solar wind speed $\left(V_{\mathrm{sw}}\right)$ : the electron path length $\left(L_{0 e}\right)$ deduced from Wind/3DP/SST data without the deposition energy loss correction (top panel), $L_{0 e}$ deduced from the deposition-energy loss corrected SST data (middle panel), and the ion path length $\left(L_{0 i}\right)$ deduced by Reames (2009a; bottom panel).

path length $L_{0 e}$ is plotted versus $V_{\mathrm{sw}}$ in the top panel of Figure 1, where $V_{\text {sw }}$ is the hourly averaged solar wind speed observed at the event onset and the solid line shows the length of the Parker spiral line at $1 \mathrm{AU}$ as a function of $V_{\mathrm{sw}}$. It can be seen that all $L_{0 e}$ data points are below the solid line, which is similar to Figure 4 of Kahler \& Ragot (2006) displaying the SST data of the 80 solar electron events they examined. In fact, the median value of $L_{0 e}$ we deduced $0.79 \pm 0.20 \mathrm{AU}$, which is consistent with $0.88 \pm$ 0.09 AU deduced by Kahler \& Ragot (2006), indicating that the lack of deposition energy loss correction of SST data may be the main reason causing an unphysical result of $L_{0 e}$ estimation.

Further, by using the deposition energy loss corrected SST data the re-calculated $L_{0 \mathrm{e}}$ value is shown in the middle panel of Figure 1, where the data points are distributed around the Parker spiral line with the median value of $L_{0 e}=1.04 \pm 0.26 \mathrm{AU}$. On the other hand, the ion path length $L_{0 i}$ deduced by Reames (2009a) is shown in the bottom panel of Figure 1, where the median value is $L_{0 i}=1.62 \pm 0.32 \mathrm{AU}$. Therefore, the $L_{0 i}$ value deduced by Reames (2009a) is still inconsistent with the $L_{0 e}$ value deduced from the deposition energy loss corrected SST data. 


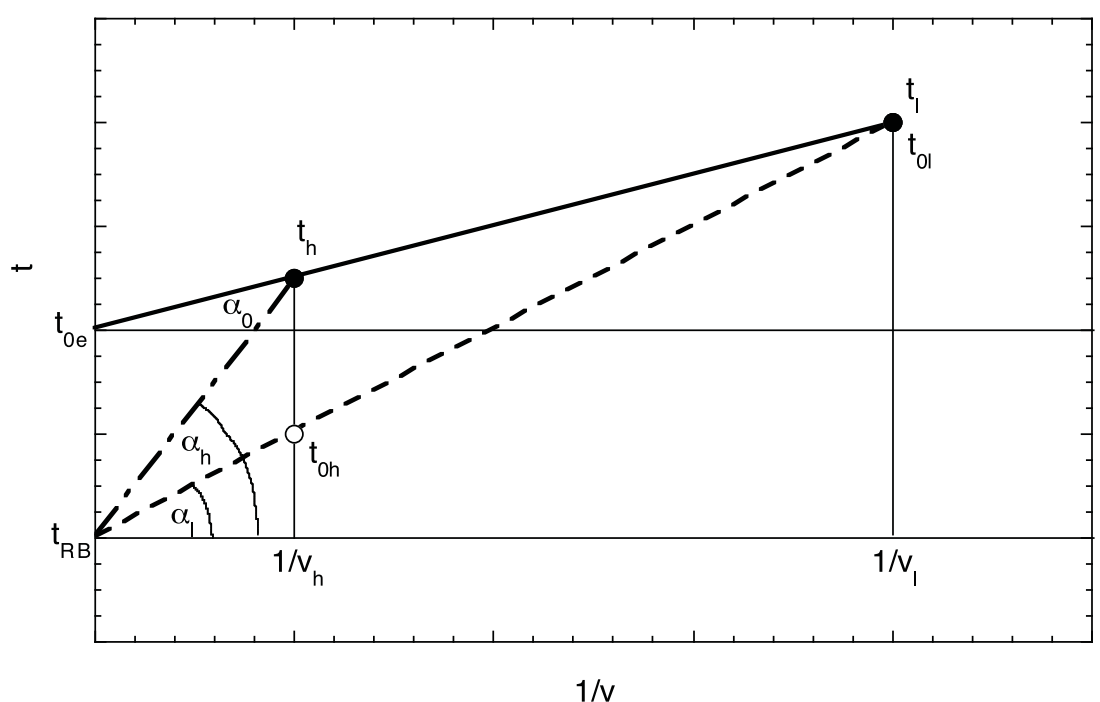

Figure 2. Calculation of electron path lengths is schematically described by plotting the electron arrival time $t$ as a function of the reciprocal of electron velocity ( $1 / v$ ). The subscripts 1 and $\mathrm{h}$ denote the low-energy and high-energy points, respectively. $\mathrm{SRT}_{e}$ deduced from the onset time analysis and the onset time of radio bursts (RBs) are denoted by $t_{0 e}$ and $t_{\mathrm{RB}}$, respectively.

\subsection{Calculation of Electron Path Lengths by Assuming $S R T_{e}=t_{R B}$}

\subsubsection{Geometrical Explanation of $L_{0 e R B}$ Deduction}

As mentioned in the Introduction, since in the SST measured energy range electron scattering increases with increasing $E_{e}$, only the IP transport of SST electrons at the lowest energy channel can be approximated to be scatter-free. Consequently, the valid $L_{0 e \mathrm{RB}}$ value should be calculated at the lowest energy channel $\left(E_{e} \sim 27 \mathrm{keV}\right)$ of SST. As $E_{e}$ increases the electron scattering status would change to become diffusive, resulting in a delay of their arrival time relative to the scatter-free transport time. That explains why the electron path length $L_{e \mathrm{RB}}$ deduced by Kahler et al. (2011b; see their Figure 3 ) is increased with increasing $E_{e}$ (i.e., decreasing $1 / v$ ).

Here we schematically describe the deduction process of both $L_{0 e}$ and $L_{0 e \mathrm{RB}}$ in Figure 2, where the electron arrival time $t$ is plotted versus the reciprocal of electron velocity $(1 / v)$ at the low-energy (l) and high-energy (h) points. In the figure the onset time analysis, which is based on the velocity dispersion relation, produces the solid line, whose slope $\left(\tan \left(\alpha_{0}\right)\right)$ is equal to the path length $L_{0 e}$. However, since in the SST energy range as $E_{e}$ increases the electron scattering status would change from scatter-free at lower energies to diffusive at higher energies, only at the low-energy point the electron arrival time $\left(t_{1}\right)$ can be approximated to the electron arrival time $\left(t_{01}\right)$ under the scatterfree transport assumption: $t_{1} \sim t_{01}$. The slope of the dashed line $\left(=\tan \left(\alpha_{1}\right)\right)$ is the path length $\left(L_{0 e \mathrm{RB}}\right)$ traveled by low-energy electrons.

On the other hand, since at the high-energy point the first arriving electrons may not experience scatter-free transport, their arrival time $t_{\mathrm{h}}$ could be delayed relative to $t_{0 \mathrm{~h}}$, the electron arrival time under the scatter-free transport assumption: $t_{\mathrm{h}}>t_{0 \mathrm{~h}}$. The path length $\left(L_{e \mathrm{RB}}\right)$ traveled by high-energy electrons is the slope $\left(\tan \left(\alpha_{\mathrm{h}}\right)\right)$ of the dot-dashed line. Since $\alpha_{0}$ is less than $\alpha_{1}$ in Figure 2, we always have $L_{0 e}<L_{0 e \mathrm{RB}}$. Also, since $\alpha_{\mathrm{h}}$ is greater than $\alpha_{1}, L_{e \mathrm{RB}}$ should increase with increasing $E_{e}$ (or decreasing $1 / v$; see Figure 3 in Kahler et al. 2011b).

For a given $E_{e}$ value, from the first arrival time $t$ of solar electrons we can calculate

$$
L_{\mathrm{eRB}}=v\left(t-t_{\mathrm{RB}}\right),
$$

where $\mathrm{RB}=$ II or III when the type II or type III RB is taken into account. Also, we have

$$
L_{0 \mathrm{eRB}}=v_{l}\left(t_{l}-t_{\mathrm{RB}}\right),
$$

where the low-energy (1) point is at the lowest energy channel $\left(E_{e} \sim 27 \mathrm{keV}\right)$ of Wind/3DP/SST electrons. For the GLE events examined, our deduced $L_{e}$ RB value at $\mathrm{RB}=\mathrm{II}$ as a function of $1 / v$ is shown in the upper panel of Figure 3. Similar to Kahler et al. (2011b), we also observe the increase of $L_{e \text { II }}$ with decreasing $1 / v$ (increasing $E_{e}$ ). In addition, among different GLE events the increasing rate of $L_{e \text { II }}$ with $E_{e}$ is different. In order to quantify the rate, we plot the deduced $L_{e \text { II }} / L_{0 e \text { II }}$ ratio as a function of $1 / v$ in the lower panel of Figure 3, from which it can be seen that the 2001 April 18 and 2003 November 2 events have the highest rate, while the 1998 May 2 and 1998 May 6 events have the lowest rate. We will explain the rate difference in Section 2.4.

\subsubsection{Comparison of $L_{O e R B}$ with $L_{O i}$}

Further, we assume that the estimated error of $L_{0 e \mathrm{RB}}$ is the difference of $L_{e \mathrm{RB}}$ values between the two lowest adjacent $E_{e}$ channels: $\Delta L_{0 e \mathrm{RB}}=L_{e \mathrm{RB}}(\sim 40 \mathrm{keV})-L_{e \mathrm{RB}}(\sim 27 \mathrm{keV})$. For the GLE events examined the $L_{0 e \mathrm{RB}} \pm \Delta L_{0 e \mathrm{RB}}$ value thus deduced for $\mathrm{RB}=\mathrm{II}$ is listed in Table 1 of this work.

Before doing the comparison between $L_{0 e \mathrm{RB}}$ and $L_{0 i}$, however, we need to check whether any significant time variation of magnetic field topologies occurred during the event period examined. By using the pitch angle spectrogram of SST electrons to perform such a check, we have observed one event (1998 August 24) in which the magnetic field topology exhibits significant variation. As shown in the upper panels of Figure 4, at the onset of the August event the electron injection is at $\mu \sim-1$, while during the later phase the injection is at $\mu \sim 1$. Because of the almost unchanged IMF direction, the $\mu$ change implies the change of IP transport routes of incident electrons during the event period. Also, in the lower panels of Figure 4 we show the time profiles of sectored counting rate data of LEMT He ions. The rate data are the counts accumulated per 5 minutes in the two wide energy ranges (2.5-5 and 5-8 $\mathrm{MeV}$ nucleon $\left.^{-1}\right)$. Additional fluctuations of recorded counts can be seen in the rate data because of varying sampling time fractions for a given 
Table 1

Solar Release Properties of the GLE Events in Solar Cycle 23

\begin{tabular}{|c|c|c|c|c|c|c|c|c|c|c|c|c|}
\hline GLE & $\begin{array}{c}\mathrm{SRT}_{i}{ }^{\mathrm{a}} \\
(\mathrm{UT})\end{array}$ & $\begin{array}{l}L_{0 i}{ }^{\mathrm{a}} \\
(\mathrm{AU})\end{array}$ & $\begin{array}{l}\mathrm{SRT}_{e} \\
(\mathrm{UT})\end{array}$ & $\begin{array}{l}L_{0 e} \\
(\mathrm{AU})\end{array}$ & Flare Class/Location $^{b}$ & $\begin{array}{c}t_{1} \\
(27 \mathrm{keV})\end{array}$ & $\begin{array}{c}t_{\mathrm{h}} \\
(65 \mathrm{keV})\end{array}$ & $\begin{array}{c}t_{\mathrm{II}}^{\mathrm{b}} \\
(\mathrm{UT})\end{array}$ & $\begin{array}{c}t_{\mathrm{III}}^{\mathrm{b}} \\
(\mathrm{UT})\end{array}$ & $\begin{array}{l}L_{0 e I I} \\
(\mathrm{AU})\end{array}$ & $\begin{array}{c}\left\langle\mu_{\text {omax }}\right\rangle \\
(65 \mathrm{keV})\end{array}$ & $\begin{array}{c}\mu_{\mathrm{m}, \mathrm{obs}} \\
(65 \mathrm{keV})\end{array}$ \\
\hline 56 & 98 May 2 13:46.7 & $1.24 \pm 0.04$ & $13: 31.6$ & $1.15 \pm 0.07$ & $1.1 / \mathrm{S} 15 \mathrm{~W} 15$ & $14: 02.1$ & $13: 52.4$ & $13: 33$ & $13: 27$ & $1.08 \pm 0.07$ & $0.79 \pm 0.02$ & $0.92 \pm 0.09$ \\
\hline 57 & 98 May $608: 03.5$ & $1.11 \pm 0.02$ & $07: 55.5$ & $1.00 \pm 0.08$ & X2.7/S11W65 & $08: 21.3$ & $08: 13.5$ & $07: 55$ & $07: 53$ & $1.01 \pm 0.07$ & $0.88 \pm 0.02$ & $0.94 \pm 0.11$ \\
\hline 58 & 98 Aug 24 22:32.1 & $1.55 \pm 0.04$ & $22: 18.8$ & $1.41 \pm 0.02$ & X1.0/N35E09 & $22: 46.2$ & $22: 37.8$ & $21: 54$ & $21: 56$ & $1.98 \pm 0.11$ & $0.42 \pm 0.06$ & $0.81 \pm 0.05$ \\
\hline 59 & 00 Jul 14 10:16.5 & $1.71 \pm 0.03$ & $10: 22.4$ & $1.09 \pm 0.09$ & X5.7/N22W07 & $10: 51.6$ & $10: 42.9$ & $10: 11$ & $10: 10$ & $1.54 \pm 0.07$ & $0.46 \pm 0.02$ & $0.86 \pm 0.07$ \\
\hline 60 & 01 Apr 15 13:47.7 & $1.59 \pm 0.01$ & $13: 51.1$ & $1.15 \pm 0.08$ & X14/S20W85 & $14: 20.4$ & $14: 12.0$ & $13: 39$ & $13: 41$ & $1.59 \pm 0.13$ & $0.52 \pm 0.02$ & $0.86 \pm 0.07$ \\
\hline 61 & 01 Apr 18 02:24.3 & $1.80 \pm 0.10$ & $02: 30.3$ & $1.08 \pm 0.07$ & $-/$ S23W 117 & $02: 57.6$ & $02: 50.4$ & 02:09 & 02:07 & $1.84 \pm 0.26$ & $0.50 \pm 0.02$ & $0.79 \pm 0.05$ \\
\hline 63 & 01 Dec 26 05:20.6 & $1.64 \pm 0.06$ & $05: 18.4$ & $1.01 \pm 0.08$ & M7.1/N08W54 & $05: 48.6$ & $05: 39.0$ & $05: 04$ & $05: 05$ & $1.69 \pm 0.10$ & $0.51 \pm 0.03$ & $0.86 \pm 0.06$ \\
\hline 64 & 02 Aug 24 01:00.1 & $2.16 \pm 0.05$ & 01:09.3 & $1.34 \pm 0.07$ & X3.1/S02W81 & $01: 45.6$ & $01: 33.0$ & $00: 53$ & $00: 53$ & $1.99 \pm 0.07$ & $0.59 \pm 0.03$ & $0.90 \pm 0.06$ \\
\hline 65 & 03 Oct 28 11:05.1 & $1.38 \pm 0.03$ & $11: 13.1$ & $0.53 \pm 0.09$ & $\mathrm{X} 17 / \mathrm{S} 20 \mathrm{E} 02$ & $11: 27.0$ & $11: 22.8$ & $10: 54$ & $10: 55$ & $1.24 \pm 0.16$ & $0.64 \pm 0.05$ & $0.76 \pm 0.07$ \\
\hline 66 & 03 Oct 29 20:55.6 & $1.75 \pm 0.09$ & $\mathrm{~N} / \mathrm{A}$ & $\mathrm{N} / \mathrm{A}$ & X10/S19W09 & $\mathrm{N} / \mathrm{A}$ & $\mathrm{N} / \mathrm{A}$ & $20: 34$ & $20: 33$ & $\mathrm{~N} / \mathrm{A}$ & $\mathrm{N} / \mathrm{A}$ & $\mathrm{N} / \mathrm{A}$ \\
\hline 67 & 03 Nov 2 17:13.8 & $2.01 \pm 0.04$ & $17: 36.5$ & $0.67 \pm 0.08$ & X8.3/S18W59 & $17: 55.8$ & $17: 47.4$ & $17: 06$ & $17: 08$ & $1.91 \pm 0.07$ & $0.29 \pm 0.03$ & $0.82 \pm 0.05$ \\
\hline 69 & 05 Jan 20 06:39.5 & $1.19 \pm 0.02$ & $06: 39.6$ & $0.96 \pm 0.08$ & X7.1/N14W61 & 07:07.2 & $06: 56.4$ & $06: 36$ & $06: 37$ & $1.10 \pm 0.07$ & $0.65 \pm 0.02$ & $0.98 \pm 0.12$ \\
\hline 70 & 06 Dec 13 02:34.0 & $1.72 \pm 0.05$ & $\mathrm{~N} / \mathrm{A}$ & $\mathrm{N} / \mathrm{A}$ & X3.4/S06W23 & $\mathrm{N} / \mathrm{A}$ & $\mathrm{N} / \mathrm{A}$ & $02: 18$ & $02: 16$ & $\mathrm{~N} / \mathrm{A}$ & $\mathrm{N} / \mathrm{A}$ & $\mathrm{N} / \mathrm{A}$ \\
\hline
\end{tabular}

Notes. The light-travel time of 8.3 minutes from the Sun to the Earth has been subtracted from the electromagnetic radiation observation time at 1 AU.

${ }^{a}$ From Reames (2009a).

b From Gopalswamy et al. (2012).

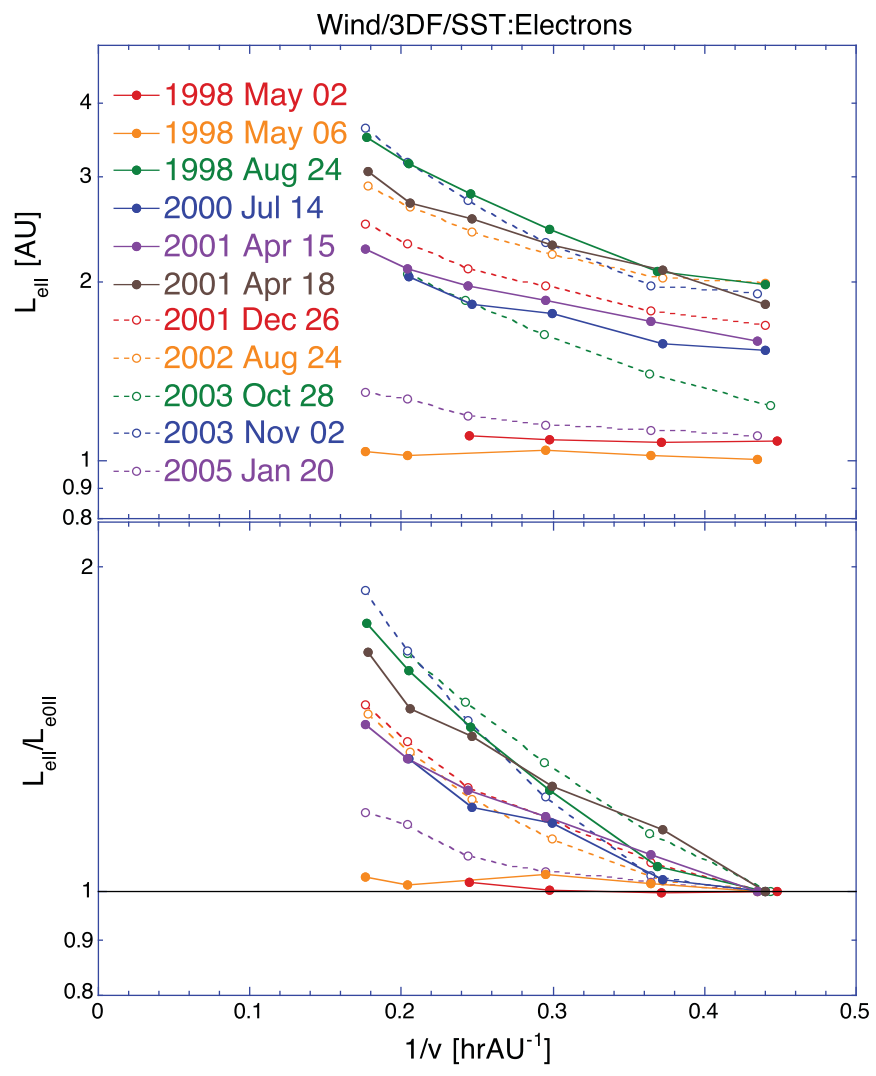

Figure 3. Assuming that the $\mathrm{SRT}_{e}$ of SST electrons is the onset time of type II RBs, for the GLE events in solar cycle 23 the deduced electron path length

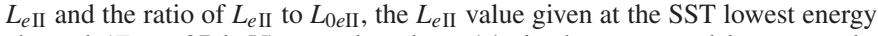
channel $\left(E_{e} \sim 27 \mathrm{keV}\right)$, are plotted vs. $1 / v$ in the upper and lower panels, respectively.

(A color version of this figure is available in the online journal.)

ion species in each 5 minute interval. Nevertheless, the rate data clearly exhibit that the first arriving $\mathrm{He}$ ions, which are delayed relative to the first arriving electrons, are at $\mu \sim 1$. Thus, both electron and $\mathrm{He}$ ion data consistently demonstrate the temporal variation of magnetic field topologies.

Therefore, the 1998 August 24 event (the open circle in Figure 5) should be rejected from the comparison between $L_{0 e \mathrm{RB}}$ and $L_{0 i}$. For the remaining 10 GLE events (the solid dot) the comparisons between $L_{0 e I I}$ and $L_{0 i}$ and between $L_{0 e I I I}$ and $L_{0 i}$ are shown in the upper and lower panels in Figure 5, respectively. The least-squares fitting results are quite good, as the linear correlation coefficients between $L_{0 e \mathrm{RB}}$ and $L_{0 i}$ are $R \sim 0.99$ in both $\mathrm{RB}=\mathrm{II}$ and III cases, indicating that the probability, by which $L_{0 e \mathrm{RB}}$ and $L_{0 i}$ are uncorrelated, is $P \sim 1 \times 10^{-7}$. However, from Figure 5 it is impossible to differentiate between $\mathrm{RB}=\mathrm{II}$ and III because of the closeness of $t_{\mathrm{II}}$ and $t_{\mathrm{III}}$. We hence calculate the weighted average of $L_{0 e \mathrm{RB}}=(0.91 \pm 0.04) L_{0 i}$ for both $\mathrm{RB}=$ II and III, i.e., $L_{0 e \mathrm{RB}} \sim L_{0 i}$ within an error range of $\pm 10 \%$.

\subsection{Correlation of $L_{\text {OeII }}$ with Properties of Non-relativistic Solar Electron Events}

\subsubsection{Correlation of $L_{0 e I I}$ with the Delay of Electron Arriving Times}

A direct use of Figure 2 is to calculate the variation of $L_{0 e I I}$ with the arriving time delay $\left(t_{\mathrm{h}}-t_{\mathrm{II}}\right)$ of high-energy electrons. From Figure 2 we have

$$
\left(t_{l}-t_{0 e}\right) v_{l}=\left(t_{\mathrm{h}}-t_{0 e}\right) v_{\mathrm{h}}
$$

Thus

$$
\begin{aligned}
L_{0 e \mathrm{II}} & =v_{l}\left(t_{l}-t_{\mathrm{II}}\right) \\
& =v_{l}\left(t_{\mathrm{h}}-t_{\mathrm{II}}\right)+\left(v_{\mathrm{h}}-v_{l}\right)\left(t_{\mathrm{h}}-t_{0 e}\right) .
\end{aligned}
$$

Since $\left(v_{\mathrm{h}}-v_{1}\right)\left(t_{\mathrm{h}}-t_{0 e}\right)$ is a smaller quantity compared to $v_{1}\left(t_{\mathrm{h}}-t_{\mathrm{II}}\right)$, we can approximate $\left(v_{\mathrm{h}}-v_{1}\right)\left(t_{\mathrm{h}}-t_{0 e}\right)$ as a constant, leading to a straight line plot of $L_{0 e \text { II }}$ versus $t_{\mathrm{h}}-t_{\mathrm{II}}$ with the slope and intercept being $v_{1}$ and $\left(v_{\mathrm{h}}-v_{1}\right)\left(t_{\mathrm{h}}-t_{0 e}\right)$, respectively. The fitting result of $E_{\mathrm{h}} \sim 65 \mathrm{keV}$ electrons is shown in the top panel of Figure 6, from which the linear correlation coefficient between $L_{0 e \mathrm{II}}$ and $t_{\mathrm{h}}-t_{\mathrm{II}}$ is $R=0.99\left(P \sim 1 \times 10^{-7}\right)$. The predicted slope $\left(v_{1}=0.038 \mathrm{AU} \mathrm{min} \mathrm{min}^{-1}\right)$ is consistent with the observed one $\left(0.040 \pm 0.002 \mathrm{AU} \mathrm{min}^{-1}\right)$. Also, from Table 1 of this work we find the averaged $\left\langle t_{\mathrm{h}}-t_{0 e}\right\rangle=0.31 \pm 0.07 \mathrm{hr}$ for the GLE events examined, and thus the predicted intercept $\left(\left(v_{\mathrm{h}}-v_{1}\right)\left(t_{\mathrm{h}}-t_{0 e}\right)\right)$ is $0.33 \pm 0.07 \mathrm{AU}$, which is consistent with the observed value $(0.27 \pm 0.08 \mathrm{AU})$. It appears that the geometric explanation reproduces the IP transport of solar electrons in the GLE event well. 
Wind/3DP/SST: Electrons
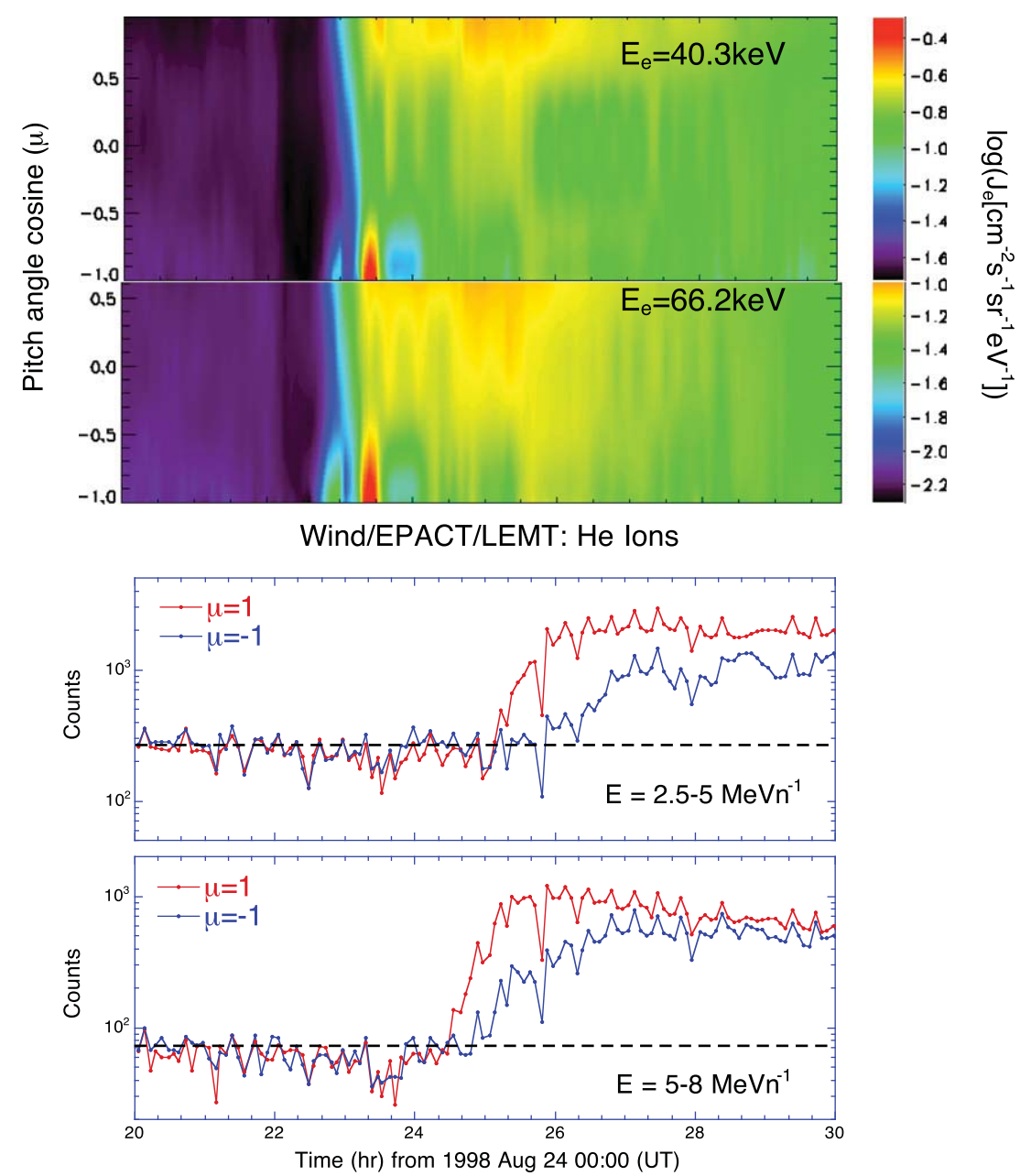

Figure 4. In the 1998 August 24 GLE event the pitch angle spectrograms of SST $E_{e} \sim 40$ and $\sim 65 \mathrm{keV}$ electrons with a 5 minute time resolution are shown in the upper panels. Also, the time profiles of sectored counting rate data of LEMT He ions are plotted in the lower panels. The rate data are the counts accumulated per 5 minutes in the two wide $E_{e}$ ranges (2.5-5 and 5-8 MeV nucleon ${ }^{-1}$ ), in which additional fluctuations of recorded counts can be seen because of varying sampling time fractions for a given ion species.

(A color version of this figure is available in the online journal.)

\subsubsection{Scattering Status of First Arriving Non-relativistic Electrons}

As mentioned in Section 2.3.2, the 2001 April 18 and 2003 November 2 events have the highest increasing rate of $L_{e \text { II }} / L_{0 e \text { II }}$ with increasing $E_{e}$, while the 1998 May 2 and 1998 May 6 events have the lowest rate. What causes the rate difference?

In Tan et al. (2011) we defined the logarithmic increasing rate of the directional electron intensity $\left(J_{e}\right)$ with increasing the pitch angle cosine $(\mu)$ of electrons,

$$
\alpha_{\mathrm{PAD}}=d \ln \left(J_{e}\right) / d \mu .
$$

It is noticeable that $\alpha_{\mathrm{PAD}}$ is a parameter characteristic of electron scattering status, because in the spherically symmetric radial diffusion model (Parker 1963) $\alpha_{\mathrm{PAD}}>3$ and $<1.5$ as deduced from the peak intensity data of particles indicate the scatterfree and diffusive transport statuses of particles, respectively (see Tan et al. 2011). While the GLE events examined may not fit the model of Parker (1963) exactly, $\alpha_{\mathrm{PAD}}$ is still a useful parameter to monitor the variation of electron scattering status.

We hence plot the time profile of $\alpha_{\mathrm{PAD}}$ for the four extreme rate events in Figure 7, where the two lower energy channels
$\left(E_{e} \sim 27\right.$ and $\left.40 \mathrm{keV}\right)$ often exhibit a slower rise of lower $\alpha_{\mathrm{PAD}}$ values, which is due to the presence of background electrons. Since background electrons usually have softer energy spectra and more isotropic PADs, their presence would result in a slower increase of smaller $\alpha_{\mathrm{PAD}}$ values at lower $E_{e}$ channels. Therefore, the maximum value of $\alpha_{\mathrm{PAD}}$ at $E_{e} \sim 65 \mathrm{keV}$ as observed at $t_{\alpha \text { PADMAX }}$ (the solid green line in Figure 7) will be used to characterize the electron scattering status at the event onset.

In Figure 7, it appears that in the 2001 April 18 and 2003 November 2 events the maximum value of $\alpha_{\mathrm{PAD}}(\sim 65 \mathrm{keV})$ is significantly lower than that in the 1998 May 2 and 1998 May 6 events, indicating that the increasing rate of $L_{e \text { II }} / L_{0 e \text { II }}$ ratios with increasing $E_{e}$ is correlated to the increase of electron scattering.

Further, from Figure 7 it can be seen that the occurrence time of $\alpha_{\mathrm{PAD}}$ maximum is delayed relative to the arrival time $t_{\mathrm{h}}$ (the green dot-dashed line). In order to explore the nature of such delay, we plot $t_{\alpha \text { PADMAX }}-t_{\text {II }}$ versus $t_{\mathrm{h}}-t_{\text {II }}$ in the second panel of Figure 6, where the least-squares fitting result between $t_{\alpha \text { PADMAX }}-\mathrm{t}_{\mathrm{II}}$ and $t_{\mathrm{h}}-t_{\mathrm{II}}$ is shown by the solid line. It can be seen that $t_{\alpha \text { PADMAX }}-t_{\mathrm{II}}$ is correlated with $t_{\mathrm{h}}-t_{\mathrm{II}}$ with the linear correlation coefficient, $R=0.83\left(P=1.6 \times 10^{-3}\right)$. 


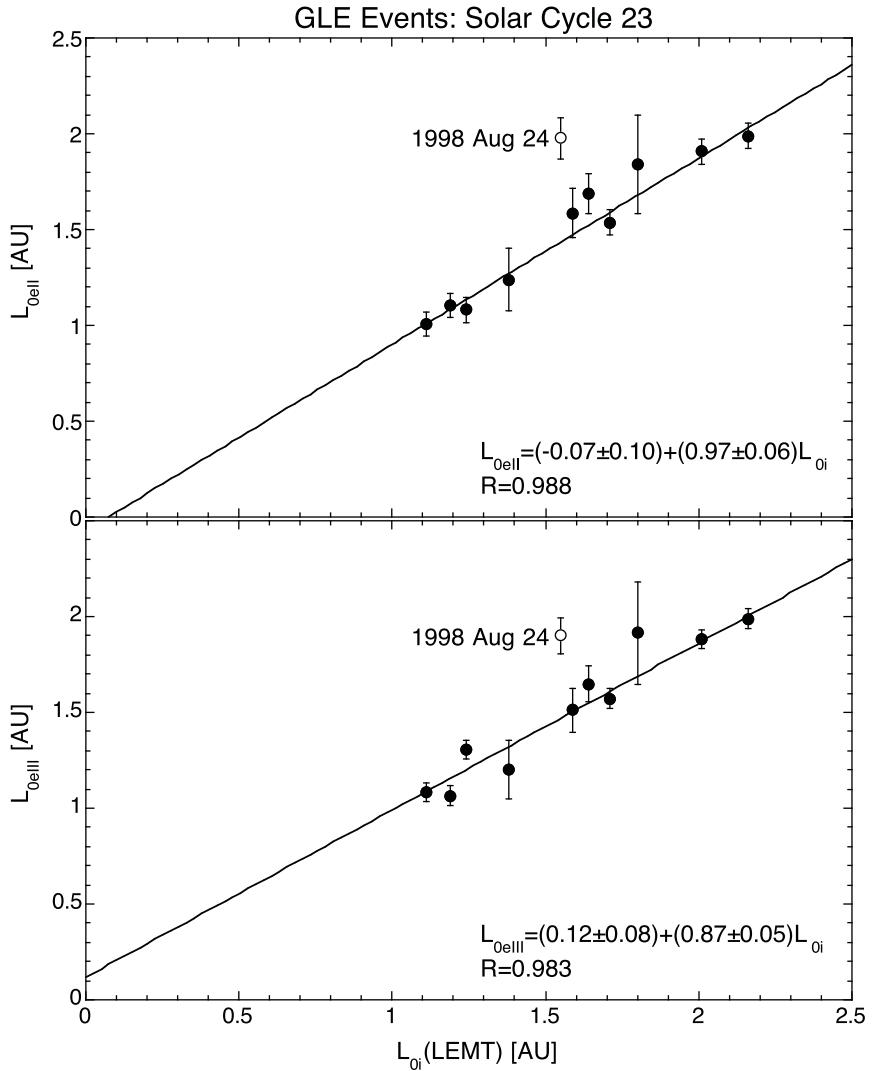

Figure 5. For the GLE events in solar cycle 23 the electron path lengths $L_{0 e I I}$ (upper) and $L_{0 e \mathrm{III}}$ (lower) deduced under the assumption of $\mathrm{SRT}_{e}=t_{\mathrm{RB}}$, where $\mathrm{RB}=\mathrm{II}$ or III, are plotted vs. the ion path length $L_{0 i}$ deduced by Reames (2009a) from the onset time analysis. The 1998 August 24 event (the open circle) has been rejected from the comparison because of the occurrence of a significant change of magnetic field topologies during the event period.

In addition, by comparing the fitting line with the dashed line showing $t_{\alpha \text { PADMAX }}=t_{\mathrm{h}}$, , we find that the mean delay of $t_{\alpha \text { PADMAX }}$ relative to $t_{\mathrm{h}}$ is $\sim 10$ minutes. The delay is the time required for enhancing the $\alpha_{\mathrm{PAD}}$ value characteristic of background electrons to that of first arriving incident electrons. In fact, assuming that at the event onset incident electrons have a step enhancement of $\alpha_{\text {PADMAX }}$, we ought to observe a gradual increase of $\alpha_{\mathrm{PAD}}$ before reaching $\alpha_{\text {PADMAX }}$ because of the "dilution" effect of background electrons. Therefore, the first arriving electrons should experience a scattering status characteristic of $\alpha_{\text {PADMAX }}$. Since among the GLE events shown in Figure 7 their $\alpha_{\text {PADMAX }}$ values are different, the IP transport status of the first arriving $E_{\mathrm{h}} \sim 65 \mathrm{keV}$ electrons may not be scatter-free.

\subsubsection{What Affects the Increasing Rate of $L_{e I I} / L_{\text {OeII }}$ with Increasing $E_{e}$ ?}

According to Equation (5), from the deduced $\alpha_{\text {PADMAX value }}$ we can calculate the averaged $\mu$ value at $\alpha_{\text {PADMAX }}$ for first arriving $E_{\mathrm{h}} \sim 65 \mathrm{keV}$ electrons,

$$
\left\langle\mu_{\text {omax }}\right\rangle=\int_{-1}^{1} \mu \exp \left(\alpha_{\text {PADMAX }} \mu\right) d \mu / \int_{-1}^{1} \exp \left(\alpha_{\text {PADMAX }} \mu\right) d \mu,
$$

whose value is listed in Table 1 . The plot of $\left\langle\mu_{\mathrm{omax}}\right\rangle$ versus $t_{\mathrm{h}}-t_{\mathrm{II}}$ is shown in the third panel of Figure 6, which exhibits the tendency that $\left\langle\mu_{\mathrm{omax}}\right\rangle$ decreases with increasing $t_{\mathrm{h}}-t_{\mathrm{II}}$. The linear correlation coefficient between $\left\langle\mu_{\text {omax }}\right\rangle$ and $t_{\mathrm{h}}-t_{\mathrm{II}}$ is $R=0.84\left(P=1.2 \times 10^{-3}\right)$. Therefore, from a joint analysis

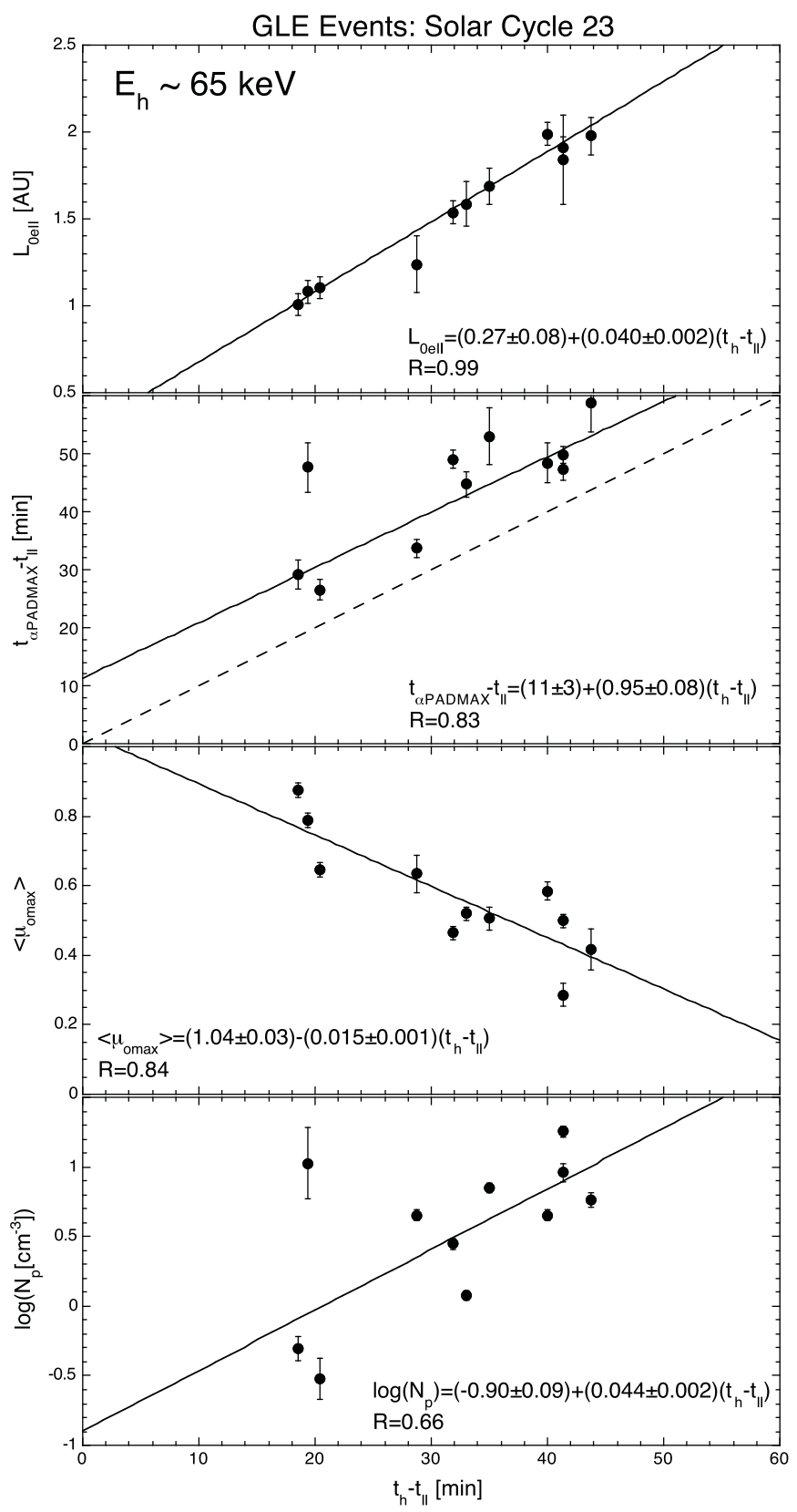

Figure 6. For $E_{\mathrm{h}} \sim 65 \mathrm{keV}$ electrons in the GLE events of solar cycle 23 the deduced $L_{0 e I I}$ value (top panel), the time difference $t_{\alpha \text { PADMAX }}-t_{\mathrm{h}}$, where $t_{\alpha \text { PADMAX }}$ and $t_{\mathrm{h}}$ are the $\alpha_{\mathrm{PAD}}$ maximum time of electrons and the onset time of type II RB, respectively (second panel), the mean $\mu$ value deduced from the locally measured $\alpha_{\mathrm{PAD}}$ maximum, $\left\langle\mu_{\mathrm{omax}}\right\rangle$ (third panel), and the solar wind density $\left(N_{\mathrm{p}}\right)$ (bottom panel) are plotted vs. $t_{\mathrm{h}}-t_{\mathrm{II}}$, where $t_{\mathrm{h}}$ is the arriving time of $E_{\mathrm{h}} \sim 65 \mathrm{keV}$ electrons.

of the top and third panels in Figure 6 we find that the GLE events with larger $L_{0 e I I}$ values would have smaller $\left\langle\mu_{\text {omax }}\right\rangle$ values, implying that due to stronger IP scattering the nonrelativistic electrons in these GLE events should experience higher increasing rates of $L_{e \text { II }} / L_{0 e \text { II }}$ with increasing $E_{e}$.

Finally, in view of the observation by Cane (2003) and Kahler et al. (2007) that the delay of arrival times of nearrelativistic electrons could be correlated with the locally measured solar wind density $\left(N_{\mathrm{p}}\right)$, we plot $1 \mathrm{hr}$ averaged $N_{\mathrm{p}}$ data from the OMNI data set versus $t_{\mathrm{h}}-t_{\mathrm{II}}$ in the bottom panel of Figure 6 , from which it can be seen that $N_{\mathrm{p}}$ and $t_{\mathrm{h}}-t_{\mathrm{II}}$ are indeed correlated with the linear correlation coefficient 
Wind/3DP/SST: Electrons
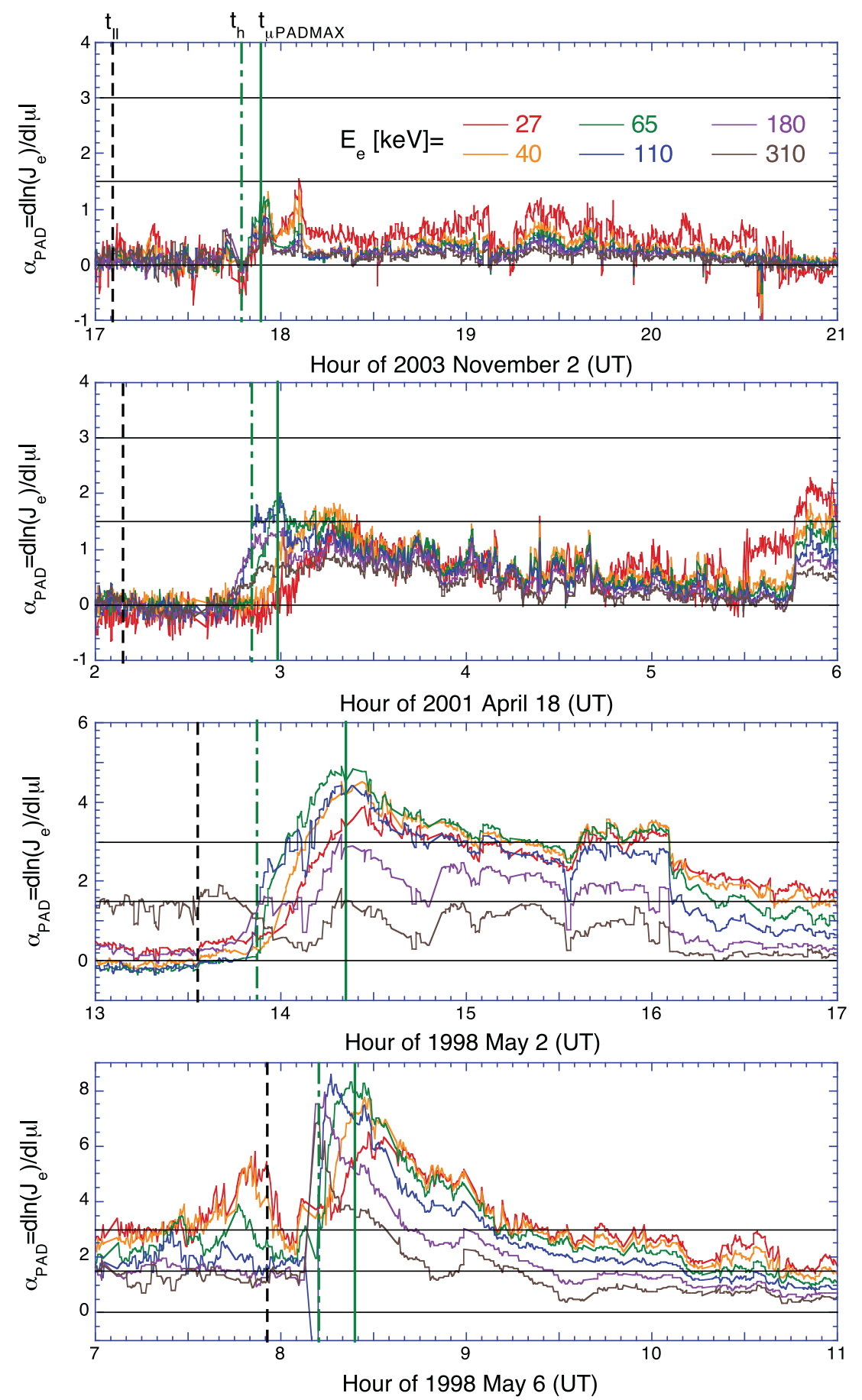

Figure 7. Time profiles of the pitch-angle-cosine increasing rate of directional electron intensity, $\alpha_{\mathrm{PAD}}$, for four GLE events, in which the rising rate of $L_{e}$ II $/ L_{0 e I I}$ with increasing $E_{e}$ is significantly deflected from the average. The $\alpha_{\mathrm{PAD}}=1.5$ and 3.0 lines denote the upper and lower limits of the diffusive and scatter-free transport regions in the spherically symmetric radial diffusion model (Parker 1963), respectively.

(A color version of this figure is available in the online journal.)

$R=0.66\left(P=2.7 \times 10^{-2}\right)$ between $\log \left(N_{\mathrm{p}}\right)$ and $t_{\mathrm{h}}-t_{\mathrm{II}}$. As explained in Section 2.3.1, the arrival time delay of $E_{e} \sim 65 \mathrm{keV}$ electrons is due to their IP scattering caused by the IMF turbulences in the local environment $(r>0.5 \mathrm{AU}$; see Tan et al. 2011; Wang et al. 2011). Since IMF turbulences are well correlated with the solar wind density fluctuations and the solar wind density itself is a reasonable proxy of its fluctuations (Richardson et al. 1998; Richardson \& Paularena 2001), the correlation between $N_{\mathrm{p}}$ and $t_{\mathrm{h}}-t_{\mathrm{II}}$ is understandable.

\subsubsection{Comparison of Locally Measured $\left\langle\mu_{\text {omax }}\right\rangle$ with the $\mu$ Value Averaged Along the Entire Electron Path Length}

As mentioned in Section 1, the change of electron scattering status should occur in the local environment because of the dominant focused transport effect of electrons at smaller $r(r<$ $0.5 \mathrm{AU}$; Tan et al. 2011; Wang et al. 2011). The local nature of the change of electron scattering status is also seen from the correlation of the solar wind density locally measured with 


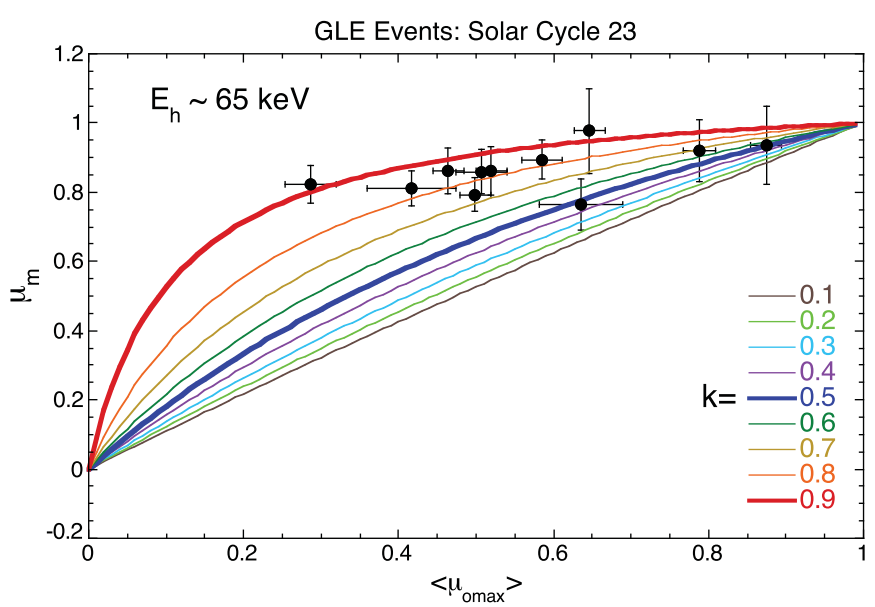

Figure 8. Mean $\mu$ value of $E_{\mathrm{h}} \sim 65 \mathrm{keV}$ electrons deduced from the delay of electron arriving times relative to the scatter-free transport time $\left(\mu_{\mathrm{m} \text {,obs }}\right.$, the solid dots $)$ is plotted vs. $\left\langle\mu_{\mathrm{omax}}\right\rangle$. Also, the predicted $\mu_{\mathrm{m}}$ values $\left(\mu_{\mathrm{m}, \mathrm{pred}}\right)$, which are based on the assumption that the electron path length $L_{0 e I I}$ can be divided into two parts comprising $k L_{0 e \text { II }}$ with $\mu=1$ and $(1-k) L_{0 e I I}$ with $\mu=\left\langle\mu_{\text {omax }}\right\rangle$ for different $k$ values are shown by color lines.

(A color version of this figure is available in the online journal.)

the electron arrival time delay as described in Section 2.4.3. Therefore, incident electrons should keep their scatter-free transport status with $\mu \sim 1$ until $r \sim 0.5 \mathrm{AU}$, beyond which the mean $\mu$ value of the first arriving electrons would change to $\left\langle\mu_{\mathrm{omax}}\right\rangle$. In order to deduce the $\mu$ value $\left(\mu_{\mathrm{m}}\right)$ averaged over $r=0-1$ AU we divide the entire path length $L_{0 \text { eII }}$ into two parts comprising $k L_{0 e \mathrm{II}}$ with $\mu=1$ and $(1-k) L_{0 e \mathrm{II}}$ with $\mu=$ $\left\langle\mu_{\text {omax }}\right\rangle$ where $k<1$. Thus $\mu_{\mathrm{m}}$ should satisfy

$$
t=t_{0}+L_{0 e \mathrm{II}} /\left(\mu_{m} v\right)
$$

where

$$
L_{0 e \mathrm{II}} /\left(\mu_{m} v\right)=k L_{0 e \mathrm{II}} / v+(1-k) L_{0 e \mathrm{II}} /\left(\left\langle\mu_{o \max }\right\rangle v\right),
$$

which results in the predicted $\mu_{\mathrm{m}}$ value

$$
\mu_{m, \text { pred }}=1 /\left(k\left(1-1 /\left\langle\mu_{o \max }\right\rangle\right)+1 /\left\langle\mu_{o \max }\right\rangle\right) .
$$

On the other hand, assuming that in Figure 2 the change of arrival times of $E_{\mathrm{h}} \sim 65 \mathrm{keV}$ electrons from $t_{0 \mathrm{~h}}$ to $t_{\mathrm{h}}$ is due to the change of their mean $\mu$ value from 1 to $\mu_{\mathrm{m}}$, we can deduce the observed $\mu_{\mathrm{m}}$ value

$$
\mu_{\mathrm{m}, \mathrm{obs}}=v_{l}\left(t_{l}-t_{\mathrm{II}}\right) /\left(v_{h}\left(t_{h}-t_{\mathrm{II}}\right)\right),
$$

which is listed in Table 1 and plotted versus $\left\langle\mu_{\mathrm{omax}}\right\rangle$ in Figure 8, where the color lines show the $\mu_{\mathrm{m} \text {,pred values estimated at }}$ different $k$ values. It can be seen that the $\mu_{\mathrm{m}, \mathrm{obs}}$ data are consistent with $k=0.7 \pm 0.2$, which is again in support of our previous conclusion (Tan et al. 2011; Wang et al. 2011, Section 2.4.3) that the change of electron scattering status should occur in the local environment $(r>0.5 \mathrm{AU})$.

\section{DISCUSSION}

\subsection{Analysis of Previous Observations}

We first analyze the apparent inconsistency between the observations of Tylka et al. (2003) and Mewaldt et al. (2003) as mentioned in Section 1. It appears that the inconsistency is probably caused by the event selection criterion used in different works. In fact, the two impulsive electron events selected by Tylka et al. (2003) should exhibit the scatter-free transport feature of the first arriving particles because of the fast rise-fast decay time profile of particle intensities observed (Lin 1974). Therefore, if the IP transport of non-relativistic electrons in the three GLE events selected by Tylka et al. (2003) is nearly scatterfree, we could expect that for all SEP events examined by Tylka et al. (2003) $L_{0 e}$ should be similar to $L_{0 i}$.

Here we check the electron transport status in the three GLE events selected by Tylka et al. (2003). Among them two events are listed in Table 1 of this work, from which it can be seen that the 1998 May 6 and 2001 April 15 events have $\mu_{\mathrm{m}, \mathrm{obs}}=0.94 \pm$ 0.11 and $0.86 \pm 0.07$, respectively, indicating that electron transport in these events is indeed close to scatter-free.

On the other hand, Mewaldt et al. (2003) classified the SEP events examined according to their ${ }^{3} \mathrm{He} /{ }^{4} \mathrm{He}$ ratios. There are four events with ${ }^{3} \mathrm{He} /{ }^{4} \mathrm{He}>0.02$ and seven events with ${ }^{3} \mathrm{He} /{ }^{4} \mathrm{He}<0.02$. Further, among the four ${ }^{3} \mathrm{He} /{ }^{4} \mathrm{He}>0.02$ events, at least three belong to the impulsive particle events (Mewaldt et al. 2003), in which near-relativistic electrons should experience scatter-free transport (Lin 1974). In addition, from Figure 3 of this work it can be seen that in the fourth (1998 May 6) event non-relativistic electrons should also experience the scatter-free transport. Thus, the $L_{0 e}$ and $L_{0 i}$ values deduced from the onset time analysis could be close to each other in all four ${ }^{3} \mathrm{He} /{ }^{4} \mathrm{He}>0.02$ events selected by Mewaldt et al. (2003).

Thus, the apparent inconsistency between Tylka et al. (2003) and Mewaldt et al. (2003) can be reduced to a notion that in the seven ${ }^{3} \mathrm{He} /{ }^{4} \mathrm{He}<0.02$ events selected by Mewaldt et al. (2003) electron transport should not be scatter-free, which sounds reasonable because of the diffusive transport feature of SEPs in these gradual SEP events (Tan et al. 2011).

Furthermore, we note that Tylka et al. (2003) only used the SST electron data at $E_{e}>100 \mathrm{keV}$, which could be due to the fact that the use of SST electron data without deposition energy loss correction would imitate an earlier arrival of lowerenergy electrons as described in Section 1. Also, the $L_{0 e}<1 \mathrm{AU}$ observed by Kahler \& Ragot (2006; see Section 3.1) could be due to the same reason. However, the difference of median $L_{0 e}$ values between EESA-H (down to $\sim 1 \mathrm{keV}$ ) and SST ( $>25 \mathrm{keV}$ ) data as observed by Kahler \& Ragot (2006) is probably caused by the change of electron scattering status with increasing $E_{e}$ (Tan et al. 2011). Since in the EESA-H events the velocity dispersion plot of electron arrival times is weighted by electron data at lower energies, where electron scattering is closer to being scatter-free, a less distorted or higher $L_{0 e}$ value should be found from EESA-H data.

\subsection{Implication of Our Observations}

Since the correlation analysis shown in Figure 5 cannot differentiate between $\mathrm{SRT}_{e}=t_{\mathrm{II}}$ and $\mathrm{SRT}_{e}=t_{\mathrm{III}}$ because of the closeness of $t_{\mathrm{II}}$ and $t_{\mathrm{III}}$, we turn to the longitude distribution of solar flares that are associated with the GLE events examined. As described in Section 1.2, solar particles accelerated in impulsive events should be confined into a narrow $\left( \pm 20^{\circ}\right)$ solar longitude cone centered at the well-connected longitude $\left(\sim \mathrm{W} 55^{\circ}-60^{\circ}\right)$. Indeed, the impulsive electron event is observed in the wellconnected longitude range $\left(\mathrm{W} 30^{\circ}\right.$ to $\mathrm{W} 90^{\circ}$; see Lin 1974; Kahler 2007), and the ${ }^{3} \mathrm{He}$-rich ion event has a similar longitude distribution (Reames 1999; Nitta et al. 2006; Tylka et al. 2012). According to Reames (1999), the longitude distribution mainly results from the variation in solar-wind speed, while the random walk of field lines also plays a smaller role. Consequently, the 


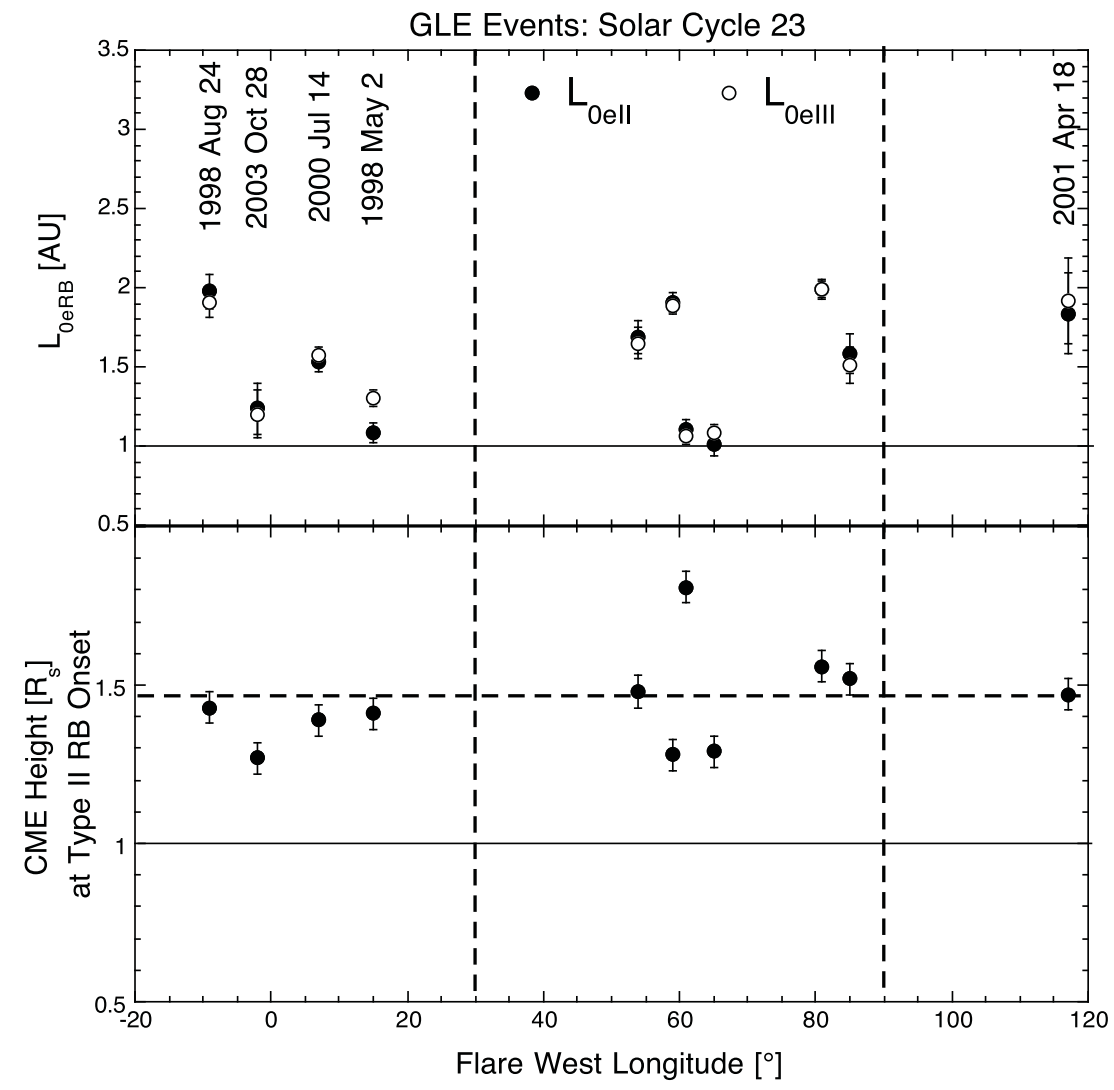

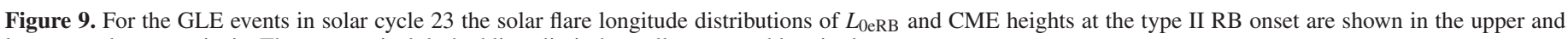
lower panels, respectively. The two vertical dashed lines limit the well-connected longitude range.

true cone of particle emission in the impulsive particle event should be smaller (Kahler et al. 2007).

The solar longitude distribution of the GLE events examined is shown in the upper panel of Figure 9, where the two vertical dashed lines limit the well-connected longitude range. What we are interested in are those GLE events outside the wellconnected longitude range, where electrons emitted from the flare acceleration process should feel difficult to reach the $1 \mathrm{AU}$ observer. We hence label these events in Figure 9. Surprisingly, we note that the 2003 October $28\left(\mathrm{E} 02^{\circ}\right)$ and 1998 May 2 $\left(\mathrm{W} 15^{\circ}\right.$ ) events have $L_{0 e \mathrm{RB}} \sim 1.2 \mathrm{AU}$, which is the length of the Parker spiral line at $1 \mathrm{AU}$ under the average solar wind speed (Figure 1), implying that the non-relativistic electrons in the two events should come from the well-connected longitude range.

We further examine the IMF data in order to explore the magnetic field topology involved in the two events. In Figure 10, the pitch angle spectrogram of Wind/3DP/SST $E_{e} \sim 40 \mathrm{keV}$ electrons and the time profile of the longitude $\left(\phi_{\mathrm{B}}\right)$ and latitude $\left(\theta_{\mathrm{B}}\right)$ of the IMF are shown in the upper and lower panels for the 1998 May 2 and 2003 October 28 events, respectively. Since a detailed analysis of the IMF topology in the May event was made in Tan et al. (2012), we first examine the magnetic field vector $\mathbf{B}$ in the May event. It can be seen from Figure 10 that at the event onset $\mathbf{B}$ is nearly Sunward with a large latitudinal component. Incident electrons are along the antisunward direction with $\mu \sim$ -1 , while reflected electrons are along the Sunward direction with $\mu>0$. Here particle reflection is due to the magnetic mirroring effect formed in the bottleneck of magnetic field lines draped around the west flank of a preceding CME (Tan et al. 1992, 2009, 2012; Bieber et al. 2002). The observed Sunward B with a large latitudinal component is consistent with the Parker spiral line distorted by the obstacle formed in the west flank of a CME (see Figure 9 in Tan et al. 2009). In addition, Figure 10 shows that the IMF configuration in the October event is similar to that in the May event: B is nearly Sunward with a large latitudinal component. The similarity implies a common origin of the field line distortion in the two events (although we do not observe the reflected electron beam in the October event). Therefore, the IMF observation is also consistent with incident electrons in the two events should come from the well-connected longitude range.

Since in the two events the event-associated flare is located at $\sim \mathrm{W}^{\circ}$, the cone of field lines that directly connect to flare particle emission must be restricted to a narrow longitude $\left( \pm \mathrm{W} 20^{\circ}\right)$ range (Reames 2002). Thus, the electrons produced in the flare acceleration process cannot directly access the 1 AU observer. As explained in Section 1.2, in order to spread to distant longitudes the particles from the flare acceleration process must be delayed and attenuated as observed by Wiedenbeck et al. (2011), which is in conflict with the observed prompt arrival of electrons with $L_{0 e \mathrm{RB}} \sim 1.2 \mathrm{AU}$.

Therefore, the solar longitude distribution and IMF topology of the GLE events examined are in favor of the CME-driven shock acceleration origin of non-relativistic electrons in the GLE events examined. Since our observations show that both nonrelativistic electrons and energetic ions could originate from the same shock acceleration process, it is understandable why both particles would have closer path lengths when they travel from their release site near the Sun to the 1 AU observer. We then estimate the CME height at type II RB onsets. From Table 1 of Gopalswamy et al. (2012) the flare longitude distribution of CME heights at the type II RB onset for the GLE events examined is shown in the lower panel of Figure 9, from which the mean height is $1.5 \pm 0.2 R_{\mathrm{S}}\left(R_{\mathrm{S}}\right.$ is the solar radius $)$. 

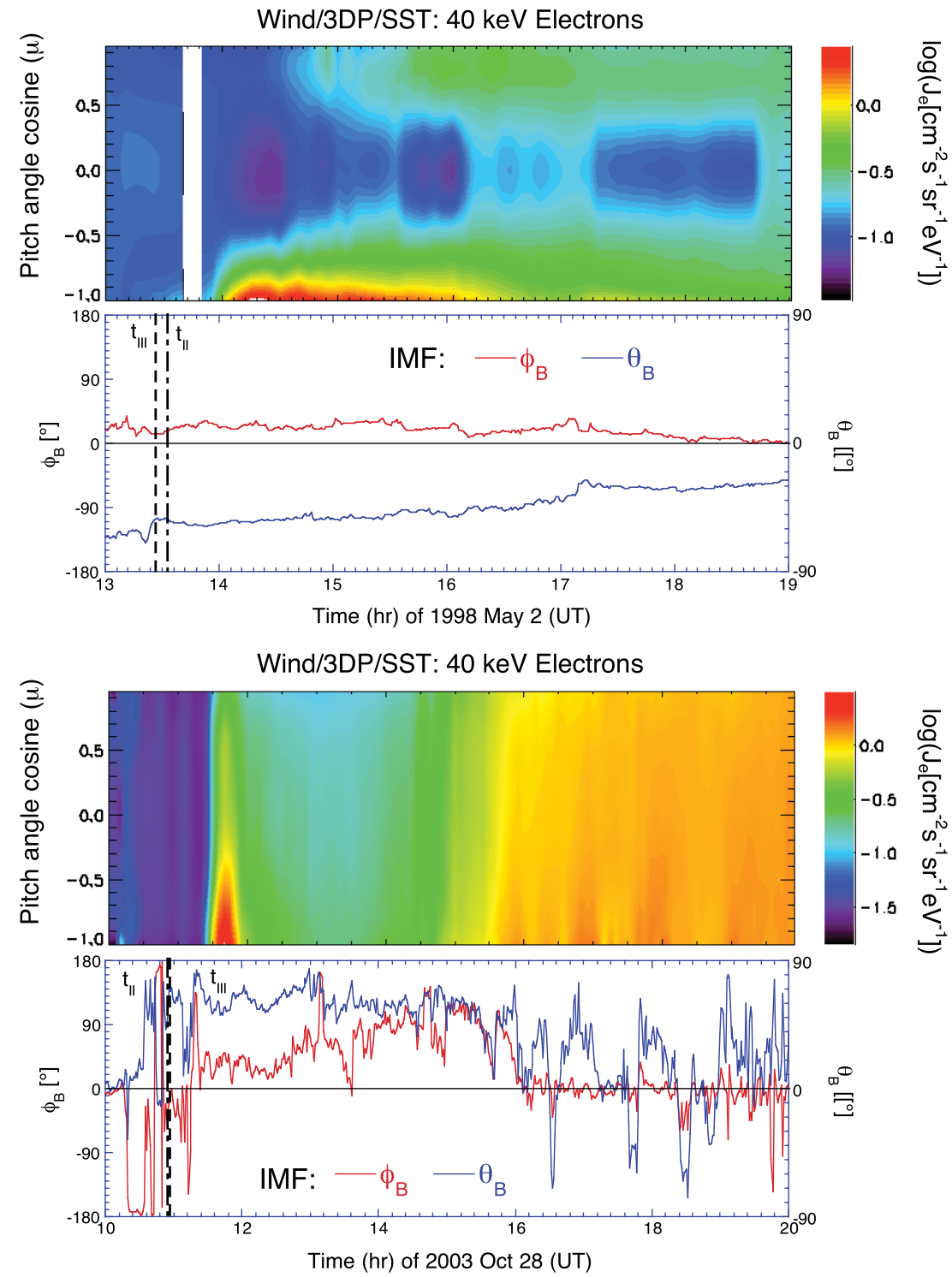

Figure 10. The pitch angle spectrogram of SST $E_{e} \sim 40 \mathrm{keV}$ electrons and the time profile of the longitude $\left(\phi_{\mathrm{B}}\right)$ and latitude $\left(\theta_{\mathrm{B}}\right)$ of the IMF are shown in the upper and lower panels for the 1998 May 2 and 2003 October 28 events, respectively.

(A color version of this figure is available in the online journal.)

Here we explain the main difference in the RB characteristics between the flare- and shock-accelerated SEP events (see the review of Reames 2013). Only along open field lines can solar electrons escape into the IPM to generate type III RBs, which are due to electron transport (Kundu 1965), independently of the acceleration source. Fast electrons streaming out ahead of slower ones would produce a "bump-on-tail" distribution function that is observed to produce Langmuir waves and subsequent radio emissions (see Thejappa et al. 2012; Graham et al. 2012, as well as the references therein). According to Wild et al. (1963), fast-driving type III RBs are the defining signature of particle acceleration by solar flares, because open field lines can be involved in the reconnection process of flare particle acceleration. On the contrary, in large gradual particle events like the GLE events examined, electrons originating from the reconnection-driven acceleration process would be trapped, because the magnetic reconnection occurs on closed field lines beneath the CME (Reames 2002).

Furthermore, CME-driven shock waves are manifested by slow-drifting type II RBs. Type II bursts are believed (see, e.g.,
Ganse et al. 2012) to be produced by electrons accelerated in the foreshock region of a shock front, where the field lines lie near the plane of the shock, perhaps even intersecting the shock surface in multiple locations. Electrons are accelerated as they drift in the electric field of a quasi-perpendicular shock. Counter-streaming electrons generate counter-streaming Langmuir waves that interact to produce the electromagnetic radiation observed as type II RBs. Since the field lines containing the electrons are soon swept downstream of the shock, the electrons may not escape. However, if they were able to find a path of outward escape, they could produce type III RBs. Shocks themselves do not produce type II RBs.

Since the spacecraft measurement of IMF cannot be used to verify the stability of magnetic flux tubes because of a small correlation scale length of the magnetic field $(\sim 0.008 \mathrm{AU}$; see Matthaeus et al. 2005), the velocity difference between faster electrons and slower ions provides a method to estimate the stability of magnetic flux tubes. Taking into account the maximum $L_{0 i}$ value found from the 2002 August 24 event (see Table 1) and the minimum energy of LEMT He ions 
$\left(\sim 1.8 \mathrm{MeV}\right.$ nucleon $\left.^{-1}\right)$, the estimated maximum stable time is $4.8 \mathrm{hr}$.

\subsection{Why Did Kahler et al. (2011a, 2011b) Not Observe Electron Path Lengths of $>3.2$ AU?}

Finally, we attempt to answer an interesting question: why did Kahler et al. (2011a, 2011b) not observe the electron path length of $>3.2 \mathrm{AU}$ in the exterior region of $\mathrm{MCs}$ under the $\mathrm{SRT}_{e}=$ $t_{\text {III }}$ assumption? In fact, from Figure 6 of this work we note a tendency that GLE events with larger $L_{0 e \text { II }}$ values have smaller $\left\langle\mu_{\text {omax }}\right\rangle$ values, implying that non-relativistic electrons in these events should experience stronger IP scattering. Extrapolating the tendency to $L_{0 e \text { II }}>3 \mathrm{AU}$, the $\left\langle\mu_{\text {omax }}\right\rangle$ value of $\sim 65 \mathrm{keV}$ electrons could be zero, indicating an isotropic PAD of electrons. Analysis (not shown here) exhibits that the variation tendency of $L_{0 e \mathrm{III}}$ is similar to that of $L_{0 e \mathrm{II}}$. Since under an isotropic PAD the diffusive transport of particles should no longer happen, in the exterior of MCs $E_{e}>65 \mathrm{keV}$ electrons would not diffusively transport along the helically wound magnetic field line to reach the 1 AU observer. Instead, the frequent occurrence of interchange reconnections between the outer fields of MCs and the surrounding solar wind fields could provide direct magnetic connections between the Sun and the 1 AU observer as suggested by Kahler et al. (2011b).

Therefore, unless electron scattering is extremely weak we cannot use $>65 \mathrm{keV}$ electrons to measure $L_{0 e I I I}$ of $>3 \mathrm{AU}$ under the $\mathrm{SRT}_{e}=t_{\mathrm{III}}$ assumption. In this sense, the 1995 October 18-20 MC event examined by Larson et al. (1997) is unusual, because in the event, electron scattering is negligible. This point can be confirmed by Figure 1 of Larson et al. (1997, also Figure 1 of Kahler et al. 2011b), which shows that the observed $L_{e \text { III }}=$ 3.2 AU is independent of $E_{e}$. Remember that (see Figure 3 of this work) an $E_{e}$-independent $L_{e \text { RB }}$ implies that the electron transport is scatter-free over the entire $E_{e}$ range. Thus, the valid result of $L_{e \text { III }}=3.2 \mathrm{AU}$ obtained by Larson et al. (1997) is guaranteed by a negligible electron scattering level over the entire $E_{e}$ range examined.

However, it can be seen from Table 1 and Figure 3 of Kahler et al. (2011b) that in the rest of the MC events they examined electron scattering is not negligible as their deduced $L_{e \text { III }}$ values exhibit a significant increase with increasing $E_{e}$. Thus from the observed data at different $E_{e}$ channels they deduced different

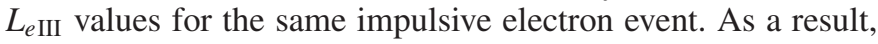
in their Table 1 only an $L_{e}$ III interval ( $L_{e}$ in the fifth column) is provided for each impulsive electron event. According to Figure 3 of this work the lower limit of the interval should be close to the $L_{0 e \text { RB }}$ value given in Section 2.3.1. For the only event common to Table 1 of Kahler et al. (2011b) and Table 1 of this work (the 1998 May 2 event) we note that the lower limit of the $L_{e \text { III }}$ interval (1.0 AU) given in Table 1 of Kahler et al. (2011b) is indeed close to the $L_{0 e \text { II }}(1.08 \pm 0.07 \mathrm{AU})$ given in Table 1 of this work, although a detailed comparison cannot be carried out without taking into account the deposition energy loss correction (Section 2.2).

It seems that significant electron scattering found inside MC could be in conflict with the general view that inside MCs the magnetic turbulence is extremely weak. However, it should be emphasized that particle scattering near the MC boundary is significantly different from that in the $\mathrm{MC}$ interior region. In fact, Torsti et al. (2004) already noted that in the 1998 May 2 $\mathrm{MC}$ event near the MC boundary the first-order anisotropy of 17-22 MeV protons is close to zero (see their Figure 1), while the

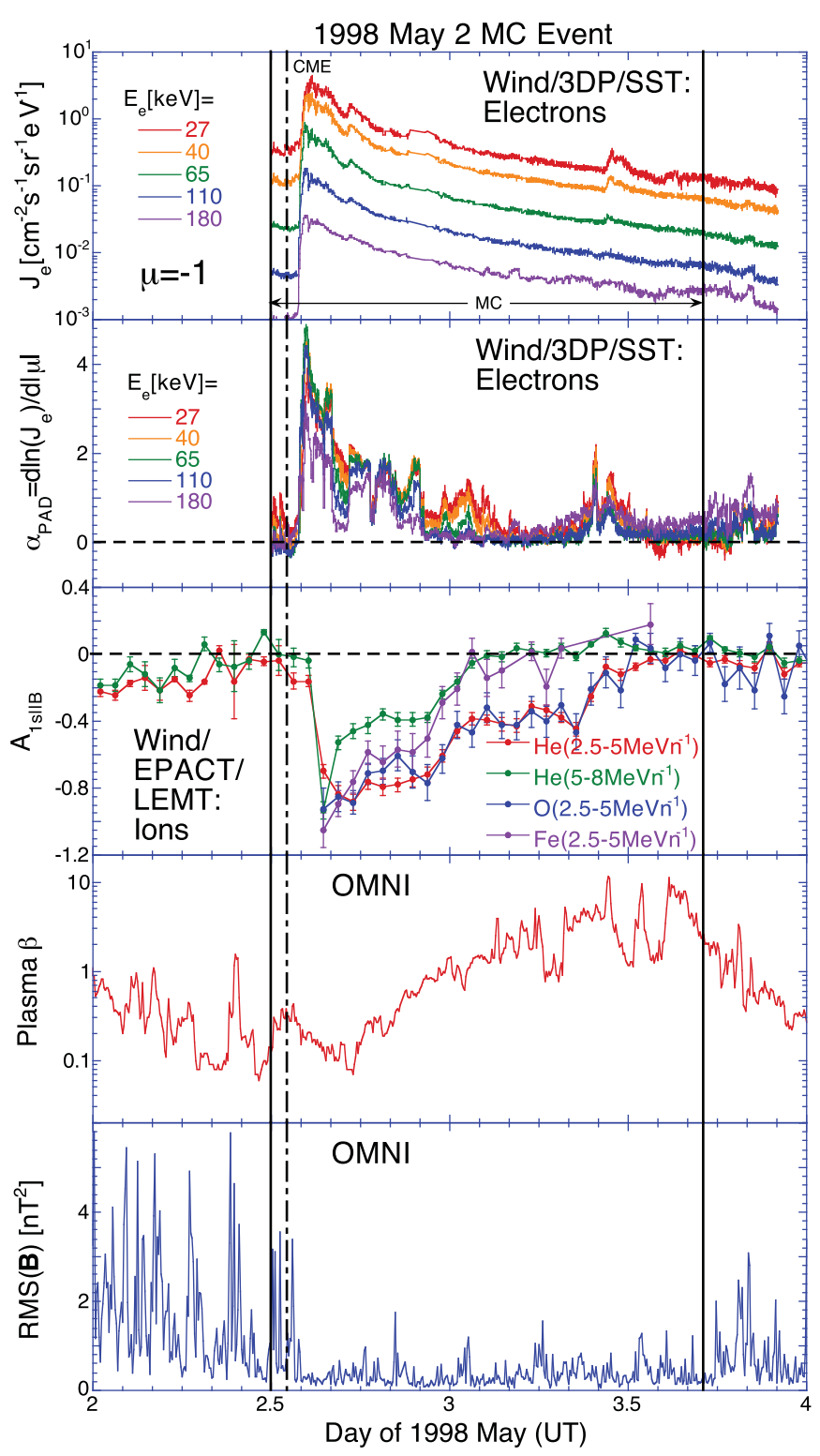

Figure 11. Time profiles of the incident electron intensity measured by SST, the $\alpha_{\text {PAD }}$ value deduced from SST electron data, the parallel to the $\mathbf{B}$ component of the first-order anisotropy vector of LEMT heavy ions in the solar wind frame $\left(A_{1 \mathrm{~s} \| \mathrm{B}}\right)$, the plasma $\beta$ value, and the root mean square deviation of the magnetic field vectors $(\operatorname{RMS}(\mathbf{B}))$ are shown from the top to bottom panels for the 1998 May $2 \mathrm{MC}$ event, respectively.

(A color version of this figure is available in the online journal.)

magnitude of ion anisotropies is anti-correlated to the intensity of magnetic fluctuations whose spatial scale is comparable to the particle Larmor radius (Kocharov et al. 2007).

Here we further analyze the details of particle scattering in the exterior region of two MC events examined in Tan et al. (2012). For the 1998 May 2 and 2002 April 21 events the time profiles of the directional intensity of incident electrons streaming away from the Sun along the IMF direction as measured by SST, the $\alpha_{\mathrm{PAD}}$ value deduced from SST data, the component parallel to $\mathbf{B}$ of the first-order anisotropy vector of LEMT heavy ions in the solar wind frame $\left(A_{1 \mathrm{~s} \| \mathrm{B}}\right)$ (see Tan et al. 2007), as well as the plasma $\beta$ (the ratio of the proton thermal energy to magnetic energy) and the root mean square deviation of magnetic field vectors $(\operatorname{RMS}(\mathbf{B}))$, both of which are from the OMNI dataset, are shown in Figures 11 and 12, respectively. 


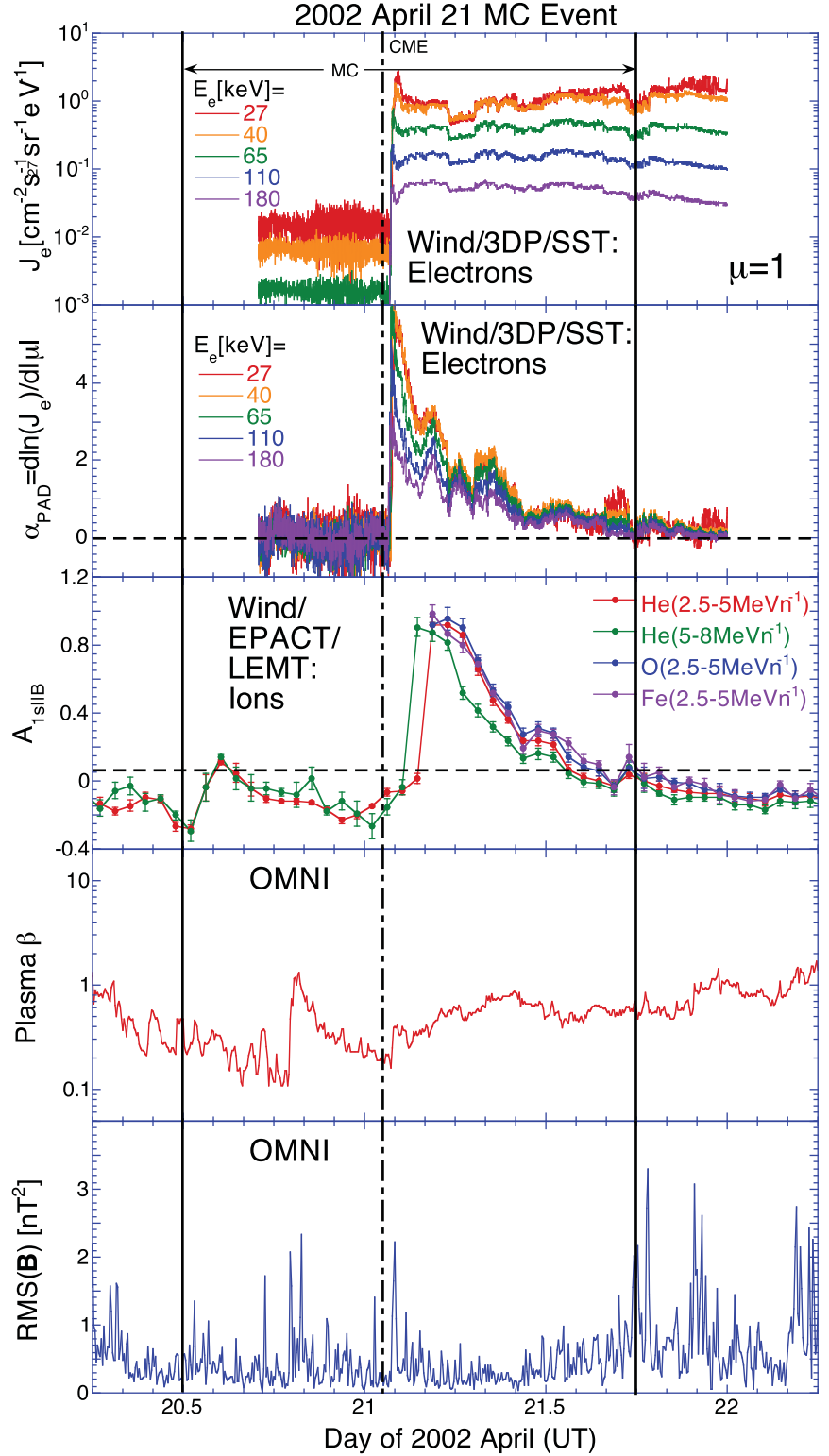

Figure 12. Same as Figure 11 but for the 2002 April 21 MC event. (A color version of this figure is available in the online journal.)

Prior to the onset of the two MC events, there existed an isotropic background electron intensity, leading to the difficulty in the identification of $\alpha_{\mathrm{PAD}}$ variations near the front boundary of MCs. Inside MCs, as time passes, $\alpha_{\mathrm{PAD}}$ gradually decreases but with significant magnitude fluctuations. At the rear boundary $\alpha_{\text {PAD }}$ is close to zero for all electron energy channels examined. In addition, the $A_{1 \mathrm{~s} \| \mathrm{B}}$ values of $\mathrm{He}, \mathrm{O}$, and $\mathrm{Fe}$ ions are also close to zero when both the front and rear boundaries are crossed. The finding that $\alpha_{\mathrm{PAD}} \sim 0$ for SST electrons and $A_{1 \mathrm{~s} \| \mathrm{B}} \sim 0$ for LEMT heavy ions implies that the magnetic field in the vicinity of the MC boundary is strongly turbulent.

We then examine the time profiles of plasma $\beta$ and $\operatorname{RMS}(\mathbf{B})$ in the exterior region of MCs. It can be seen that around the front boundary of MCs there exist a gradient of $\beta$ and an enhancement of $\operatorname{RMS}(\mathbf{B})$, which could affect particle scattering through the following processes.

1. According to Burlaga (1991), the gradient of $\beta$ at the MC boundary should produce a ballooning instability (Strauss
1989), which could cause the transport of turbulence, leading to a broadened boundary layer with strong turbulence. While this mechanism has not been investigated in detail, Burlaga et al. (2001) pointed out that there might be a broader, turbulent transition at the front boundary, and a tail or wake at the rear boundary.

2. Leamon et al. (1998) examined the anisotropy of IMF fluctuations inside MCs. They found that near the center of MCs magnetic fluctuations are more nearly transverse to the mean field than that in the open field line case. Also, the wave vector is oriented at larger angles relative to the mean field vector. Therefore, Smith et al. (1999) pointed out that the weak scattering inside MCs is not only caused by the decreased magnitude of magnetic fluctuations, but also due to the orientation of the wave vector that is especially ineffective at scattering particles. Since the extremely transverse magnetic fluctuations only show up in the center of MCs, the magnetic fluctuations near the MC boundary could scatter particles.

Therefore, there should be sufficient electron scattering existing in the exterior region of MCs to support the extrapolation of $\left\langle\mu_{\text {omax }}\right\rangle$ and $L_{0 e \text { II }}$ to higher $t_{\mathrm{h}}-t_{\mathrm{II}}$ values in Figure 6, leading to a scenario that $\left\langle\mu_{\text {omax }}\right\rangle$ could be close to zero when $L_{0 e \text { II }}>3$ AU.

\section{SUMMARY}

In this work, we have examined the Wind/3DP/SST electron data and Wind/EPACT/LEMT ion data to investigate the properties of GLE events during solar cycle 23. Our main findings are as follows.

1. Under the $\mathrm{SRT}_{e}=t_{\mathrm{RB}}$ assumption with $\mathrm{RB}=\mathrm{II}$ or III, the deduced path length $L_{e \mathrm{RB}}$ of electrons is found to increase with increasing $E_{e}$, indicating that at higher energies the first arriving electrons may not experience scatter-free transport.

2. The increasing rate of $L_{e \mathrm{RB}} / L_{0 e \mathrm{RB}}$, where $L_{0 e \mathrm{RB}}$ is the $L_{e \mathrm{RB}}$ value measured at the lowest energy of SST, is correlated with the pitch angle distribution (PAD) of peak electron intensities locally measured, with a higher rate corresponding to a broader PAD. The correlation implies that the broadening of electron PADs at larger $L_{0 e \mathrm{RB}}$ events is due to a stronger IP scattering experienced by electrons in the local environment ( $r>0.5 \mathrm{AU})$.

3. For the GLE events examined the correlation between $L_{0 e \mathrm{RB}}$ and $L_{0 i}$ are quite good for both $\mathrm{RB}=\mathrm{II}$ and III. The linear correlation coefficient is $R \sim 0.99$, indicating that the probability of $L_{0 e \mathrm{RB}}$ and $L_{0 i}$ being uncorrelated is $P \sim 1 \times$ $10^{-7}$. However, based on the correlation examination it is impossible to differentiate between $\mathrm{RB}=\mathrm{II}$ and III because of the closeness of $t_{\mathrm{II}}$ and $t_{\mathrm{III}}$. The weighted averaged $L_{0 e \mathrm{RB}}=(0.91 \pm 0.04) L_{0 i}$ is deduced from both $\mathrm{RB}=$ II and III.

4. The solar longitude distribution and IMF topology of the GLE events examined are in favor of the CME-driven shock acceleration origin of the non-relativistic electrons observed.

5. The velocity difference between faster electrons and slower ions provides a method to estimate the stability of magnetic flux tubes. From the GLE events examined the estimated maximum stable time of magnetic flux tubes is $4.8 \mathrm{hr}$.

We gratefully acknowledge data provided by the NASA/Space Physics Data Facility (SPDF)/CDAWeb and 
Wind/3DP Data Center. Also, we thank K. Ogilvie, R. Lin, and A. Szabo for their support of this work, and the anonymous reviewer for his/her valuable comments. The presented work has received funding from the European Union Seventh Framework Programme (FP7/2007-2013) under grant agreement No. 263252 [COMESEP]. Also, D.V.R. is supported in part by NASA grant NNX08AQ02G. C.K.N. is supported in part by NASA grant NNX09AU98G. L.W. is supported in part by NASA grant NNX08AE34G.

\section{REFERENCES}

Berger, M. J., Seltzer, S. M., Chappell, S. E., Humphreys, J. C., \& Motz, J. W. 1969, NucIM, 69, 181

Bieber, J. W., Dröge, W., Evenson, P. A., et al. 2002, ApJ, 567, 622

Bougeret, J.-L., Kaiser, M. L., Kellogg, P. J., et al. 1995, SSRv, 71, 231

Burlaga, L. F. 1991, in Physics and Chemistry in Space: Space and Solar Physics,

Vol. 21, Physics of the Inner Heliosphere II, ed. R. Schwenn \& E. Marsch (Berlin: Springer), 1

Burlaga, L. F., Skoug, R. M., Smith, C. W., et al. 2001, JGR, 106, 20957

Cane, H. V. 2003, ApJ, 598, 1403

Cane, H. V., Richardson, I. G., \& von Rosenvinge, T. T. 2010, JGR, 115, A08101

Chollet, E. E., \& Giacalone, J. 2011, ApJ, 728, 64

Cliver, E. W. 2008, in IAU Symp. 257, Universal Heliophysical Processes, ed. N. Gopalswamy \& D. F. Webb (Cambridge: Cambridge Univ. Press), 401

Ganse, U., Kilian, P., Vainio, R., \& Spanier, F. 2012, SoPh, 280, 551

Gopalswamy, N., Xie, H., Yashiro, S., et al. 2012, SSRv, 171, 23

Gosling, J. T., Skoug, R. M., \& McComas, D. J. 2003, GeoRL, 30, 1967

Graham, D. B., Cairns, I. H., Malaspina, D. M., \& Ergun, R. E. 2012, ApJL, 753, L18

Haggerty, D. K., \& Roelof, E. C. 2002, ApJ, 579, 841

Haggerty, D. K., Roelof, E. C., \& Simnett, G. M. 2003, AdSpR, 32, 2673

He, H.-Q., \& Wan, W. 2012, ApJ, 747, 38

He, H.-Q., Qin, G., \& Zhang, M. 2011, ApJ, 734, 74

Jokipii, J. R. 1971, RvGeo, 9, 27

Jokipii, J. R., \& Parker, E. N. 1969, ApJ, 155, 777

Kahler, S. W. 2007, SSRv, 129, 359

Kahler, S. W., Aurass, H., Mann, G, \& Klassen, A. 2007, ApJ, 656, 567

Kahler, S. W., Haggerty, D. K., \& Richardson, I. G. 2011a, ApJ, 736, 106

Kahler, S. W., Krucker, S., \& Szabo, A. 2011b, JGR, 116, A01104

Kahler, S. W., \& Ragot, B. R. 2006, ApJ, 646, 634

Kocharov, L., Saloniemi, O., Torsti, J., Kovaltsov, G., \& Riihonen, E. 2007, ApJ, 654,1121

Krucker, S., Kontar, E. P., Christe, S., \& Lin, R. P. 2007, ApJL, 663, L109

Krucker, S., Larson, D. E., Lin, R. P., \& Thompson, B. J. 1999, ApJ, 519, 864

Kundu, M. R. 1965, Solar Radio Astronomy (New York: Wiley-Interscience)

Larson, D. E., Lin, R. P., McTiernan, J. M., et al. 1997, GeoRL, 24, 1911

Leamon, R. J., Smith, C. W., \& Ness, N. F. 1998, JGR, 25, 2505
Lin, R. P. 1974, SSRv, 16, 189

Lin, R. P. 1985, SoPh, 100, 537

Lin, R. P., Anderson, K. A., Ashford, S., et al. 1995, SSRv, 71, 125

Matthaeus, W. H., Dasso, S., Weygand, J. M., et al. 2005, PhRvL, 95, 231101

Mazur, J. E., Mason, G. M., Dwyer, J. R., et al. 2000, ApJL, 532, L79

Mewaldt, R. A., Cohen, C. M. S., Haggerty, D. K., et al. 2003, in Proc. 28th Int. Cosmic Ray Conf., Vol. 6, ed. T. Kajta, Y. Asaoka, A. Kawachi et al. (Tokyo: Universal Academy Press, Inc.), 3313

Ng, C. K., Reames, D. V., \& Tylka, A. J. 2003, ApJ, 591, 461

Nitta, N. V., Reames, D. V., De Rosa, M. L., et al. 2006, ApJ, 650, 438

Parker, E. N. 1963, Interplanetary Dynamical Process (New York: WileyInterscience)

Reames, D. V. 1999, SSRv, 90, 413

Reames, D. V. 2002, ApJL, 571, L63

Reames, D. V. 2009a, ApJ, 693, 812

Reames, D. V. 2009b, ApJ, 706, 844

Reames, D. V. 2013, SSRv

Richardson, J. D., Dashevskiy, F., \& Paylarena, K. I. 1998, JGR, 103, 14,619

Richardson, J. D., \& Paularena, K. I. 2001, JGR, 106, 239

Rouillard, A. P., Sheeley, Jr., N. R., Tylka, A., et al. 2012, ApJ, 752, 44

Saiz, A., Evenson, P., Ruffolo, D., \& Bieber, J. W. 2005, ApJ, 626, 1131

Smith, C. W., Leamon, R. J., Ness, N. F., et al. 1999, in Proc. 26th Int. Cosmic Ray Conf., ed. by D. Kieda, M. Salamon, \& B. Dingus (Salt Lake City, UT: Univ. of Utah), 7, 4809

Strauss, H. R. 1989, GeoRL, 16, 219

Tan, L. C., Malandraki, O. E., Reames, D. V., et al. 2012, ApJ, 750, 146

Tan, L. C., Mason, G. M., Lee, M. A., et al. 1992, JGR, 97, 1597

Tan, L. C., Reames, D. V., \& Ng, C. K. 2007, ApJ, 661, 1297

Tan, L. C., Reames, D. V., Ng, C. K., Saloniemi, O., \& Wang, L. 2009, ApJ, 701,1753

Tan, L. C., Reames, D. V., Ng, C. K., Shao, X., \& Wang, L. 2011, ApJ, 728,133

Thejappa, G., MacDowall, R. J., Bergamo, M., \& Papadopoulos, K. 2012, ApJL, 747, L1

Torsti, J., Riihonen, E., \& Kocharov, L. 2004, ApJL, 600, L83

Turner, R. E. 2006, in Solar Eruptions and Energetic Particles, ed. N. Gopalswamy et al. (Geophys. Monogr. Ser., Vol. 165; Washington, DC: AGU), 367

Tylka, A. J., Cohen, C. M. S., Diertrich, W. F., et al. 2003, in Proc. 28th Int. Cosmic Ray Conf., Vol. 6, ed. T. Kajta, Y. Asaoka, A. Kawachi et al. (Tokyo: Universal Academy Press, Inc.), 3305

Tylka, A. J., \& Lee, M. A. 2006, ApJ, 646, 1319

Tylka, A. J., Malandraki, O. E., Dorrian, G., et al. 2012, SoPh

von Rosenvinge, T. T., Barbier, L. M., Karsch, J., et al. 1995, SSRv, 71, 155

Wang, L., Lin, R. P., \& Krucker, S. 2011, ApJ, 727, 121

Wang, L., Lin, R. P., Krucker, S., \& Gosling, J. T. 2006, GeoRL, 33, L03106

Wiedenbeck, M. E., Mason, G. M., Cohen, C. M. S., et al. 2011, in Proc. 32nd Int. Cosmic Ray Conf., Vol. 10, ed. Y. Tan et al. (Beijing: Institute of High-Energy Physics), 10, 205

Wild, J. P., Smerd, S. F., \& Weiss, A. A. 1963, ARA\&A, 1, 291 